DESY 97-126

hep-ph/9707251

July 1997

\title{
An analysis of two-body non-leptonic $B$ decays involving light mesons in the Standard Model
}

\author{
A. Ali and C. Greub \\ Deutsches Elektronen Synchrotron DESY, Hamburg, Germany
}

\begin{abstract}
We report a theoretical analysis of the exclusive non-leptonic decays of the $B^{ \pm}$and $B^{0}$ mesons into two light mesons, some of which have been measured recently by the CLEO collaboration. Our analysis is carried out in the context of an effective Hamiltonian based on the Standard Model (SM), using next-to-leading order perturbative QCD calculations. We explicitly take into account the $O\left(\alpha_{s}\right)$ penguin-loop diagrams of all four-Fermi operators and the $O\left(\alpha_{s}\right)$ tree-level diagram of the chromomagnetic dipole operator, and give a prescription for including their effects in non-leptonic two-body decays. Using a factorization ansatz for the hadronic matrix elements, we show that existing data, in particular the branching ratios $\mathcal{B}\left(B^{ \pm} \rightarrow \eta^{\prime} K^{ \pm}\right)$, $\mathcal{B}\left(B^{ \pm} \rightarrow \pi^{ \pm} K^{0}\right), \mathcal{B}\left(B^{0}\left(\overline{B^{0}}\right) \rightarrow \pi^{\mp} K^{ \pm}\right)$, and $\mathcal{B}\left(B^{ \pm} \rightarrow \omega h^{ \pm}\right)\left(h^{ \pm}=\pi^{ \pm}, K^{ \pm}\right)$, can be accounted for in this approach. Thus, theoretical scenarios with a substantially enhanced Wilson coefficient of the chromomagnetic dipole operator (as compared to the SM) and/or those with a substantial color-singlet $c \bar{c}$ component in the wave function of $\eta^{\prime}$ are not required by these data. We predict, among other decay rates, the branching ratios for the decays $B^{0}\left(\overline{B^{0}}\right) \rightarrow \pi^{ \pm} \pi^{\mp}$ and $B^{ \pm} \rightarrow \pi^{0} \pi^{ \pm}$, which are close to the present experimental limits. Implications of some of these measurements for the parameters of the CKM matrix are presented.
\end{abstract}

(Submitted to Physical Review D) 


\section{Introduction}

Recently, the CLEO collaboration has reported first measurements in a number of exclusive decays, $B^{ \pm} \rightarrow \eta^{\prime} K^{ \pm}, B^{ \pm} \rightarrow \pi^{ \pm} K^{0}, B^{0}\left(\overline{B^{0}}\right) \rightarrow \pi^{\mp} K^{ \pm}, B^{ \pm} \rightarrow \omega h^{ \pm}\left(h^{ \pm}=\pi^{ \pm}, K^{ \pm}\right), B^{ \pm} \rightarrow$ $K^{0} h^{ \pm}, B^{ \pm} \rightarrow \pi^{0} h^{ \pm}, B^{0}\left(\overline{B^{0}}\right) \rightarrow h^{ \pm} \pi^{\mp}$, and the inclusive decay $B^{ \pm} \rightarrow \eta^{\prime}+X$ [1, 2, 3], which involve the so-called QCD penguins. In addition, a number of related decays such as $B^{0}\left(\overline{B^{0}}\right) \rightarrow$ $\pi^{\mp} \pi^{ \pm}$and $B^{ \pm} \rightarrow \pi^{ \pm} \pi^{0}$ are on the verge of measurement [3]. On the theoretical side, considerable effort has gone into studies of non-leptonic weak decays in terms of estimating decay rates [4] ] - [13 and the inherent direct and indirect CP asymmetries [10], [14 - 22]. Since the first measurements of the above-mentioned decays, theoretical interest in this subject has surged and recent literature is rife with all kinds of interesting interpretations of data, both within and beyond the SM [23] - [29]. Of these, the decay mode $B^{ \pm} \rightarrow \eta^{\prime} K^{ \pm}$is conspicuous due to its reported high branching ratio $\mathcal{B}\left(B^{ \pm} \rightarrow \eta^{\prime} K^{ \pm}\right)=\left(7.1_{-2.1}^{+2.5} \pm 0.9\right) \times 10^{-5}$ [3] .

The standard theoretical framework to study non-leptonic $B$ decays is based on the effective Hamiltonian approach, which allows to separate the short- and long-distance contributions in these decays using the Wilson operator product expansion [30]. QCD perturbation theory is then used in deriving the renormalization group improved short-distance contributions [31]. This program has now been carried out up to and including the next-to-leading order terms [32, 33], but the long-distance part in the two-body hadronic decays $B \rightarrow M_{1} M_{2}$ involves the transition matrix elements $\left\langle M_{1} M_{2}\left|O_{i}\right| B\right\rangle$ at a typical hadronic scale, where $O_{i}$ is an operator in the effective Hamiltonian (see below). Calculating these matrix elements from first principle is a true challenge in theory which remains to be met. In view of this, a number of approximate schemes has been put forward. The one we use here is based on the idea of factorization [34, 35], in which the final state interactions (FSI) are assumed absent, and hence the hadronic matrix elements in the decay $B \rightarrow M_{1} M_{2}$ factorize into a product of two comparatively more tractable matrix elements. These are then taken either from data or calculated in well defined theoretical contexts, such as QCD sum rules and potential models [4, 13], [36]- [40]. This framework does remarkably well in accounting for non-leptonic two-body $B$ decays involving the current-current operators $O_{1,2}^{c}$ औ, [] (see section 2 for definition). Recent analyses have shown that data on twobody non-leptonic $B$ decays on the so-called heavy-to-heavy transitions, such as $B \rightarrow\left(D, D^{*}\right) h$, $B \rightarrow\left(D_{s}, D_{s}^{*}\right) D, B \rightarrow J / \psi h$ (with $h$ being a light hadron), can be described in terms of two phenomenological parameters, $a_{1}$ and $a_{2}$ [4], whose values seem to be universal [41, 13]. Techniques based on HQET 42 allow in some limited cases to "derive" such factorization properties 43] and yield results which are in agreement with data.

Motivated by the phenomenological success of factorization in the heavy-to-heavy nonleptonic $B$ decays, we would like to pursue further this framework in the domain of the socalled heavy-to-light transitions, $B \rightarrow h_{1} h_{2}$, where $h_{1}$ and $h_{2}$ are light hadrons. The recently measured $B$ decays [1, 2, 3] belong to this category and they should be analyzed on their own, without prejudice about the suggested values of the effective parameters from the heavy-toheavy transitions. The decays $B \rightarrow h_{1} h_{2}$ in most cases involve mixing among the currentcurrent, QCD-penguin and the chromomagnetic operators. Our hope is that once perturbative QCD corrections are taken into account, these decays may allow themselves to be described in terms of a few phenomenological parameters. Related work along these lines concerning QCD penguins in non-leptonic $B$ decays has been done prior to this analysis [8, 10], which we make use of here, improve upon and extend. 
Our analysis is based on the following three main ingredients:

- We work at next-to-leading logarithmic (NLL) precision, taking into account the $O\left(\alpha_{s}\right)$ one-loop penguin-type diagrams of all four-Fermi operators in the effective Hamiltonian and some process-independent parts of the vertex correction diagrams associated with these four-Fermi operators. We also take into account the effect of the $O\left(\alpha_{s}\right)$ tree-level matrix element associated with the chromomagnetic dipole operator via the process $b \rightarrow$ $s g \rightarrow s \bar{q}^{\prime} q^{\prime}$.

- To calculate the hadronic matrix elements, we propose a simple factorization ansatz which allows to include the effects of the $O\left(\alpha_{s}\right)$ matrix elements just discussed above.

- In calculating $B$ decays involving an $\eta^{\prime}$ or $\eta$ meson, such as $B^{ \pm} \rightarrow \eta^{\prime} K^{ \pm}$and $B^{ \pm} \rightarrow \eta K^{ \pm}$, we include the contribution from the decay $b \rightarrow s(c \bar{c}) \rightarrow s\left(\eta, \eta^{\prime}\right)$ [44, 45]. The required decay constants and mixing parameters are estimated using data on the radiative decays $J / \psi \rightarrow \eta_{c} \gamma, \eta^{\prime} \gamma, \eta \gamma$ and the two-photon decays of the $\eta, \eta^{\prime}$, and $\eta_{c} 46$. Concerning $\left(\eta, \eta^{\prime}\right)$ mixing, we discuss both the conventional (one mixing-angle) formalism [47, 48] and the one involving two mixing-angles in this sector, which is suggested by the $1 / N_{c}$-improved $U(3) \otimes U(3)$ chiral perturbation theory framework 449, 50. Since the latter formalism is also favoured by a recent phenomenological analysis [51] of the data on the $\eta \gamma$ and $\eta^{\prime} \gamma$ form factors [52]- [55], we use it in our estimates for $B$ decays involving $\eta^{\prime}$ and $\eta$-meson.

We would like to make a number of remarks pointing out the overlaps and differences with earlier analyses and explaining our factorization ansatz.

Concerning the QCD-perturbative part, we note that our calculations come close to the derivation given in [10] but are more complete as far as the NLL contribution is concerned. We find that the NLL improvements implemented by us reduce the scale dependence in various non-leptonic decay rates. This result is in line with what has been demonstrated in the radiative decays $B \rightarrow X_{s}+\gamma$ in the same accuracy [56]. Further, the complete NLL contribution is important numerically, both compared to the leading order result and the NLL result obtained by keeping only the charm penguin contributions from the operators $O_{1,2}^{c}$. We show this quantitatively in the context of the branching ratio $\mathcal{B}\left(B^{ \pm} \rightarrow K \pi^{ \pm}\right)$, comparing it with the estimates of the same based on keeping only the $O_{1,2}^{c}$ penguins [2\&].

Concerning the second point noted above, we remark that our factorization prescription introduces just one free parameter, called $\xi$, which is supposed to compensate for the neglect of color octet-octet contribution in evaluating the hadronic matrix elements in the heavy-tolight sector $B \rightarrow h_{1} h_{2}$. This modifies the strength of the effective coefficients $a_{1}, \ldots, a_{6}$ from their perturbatively calculated values (see section 3). Clearly, this is the simplest ansatz and may have to be modified eventually as more precise data on heavy to light $B$ decays become available.

We discuss the last point mentioned above concerning the decays $B^{ \pm} \rightarrow \eta^{\prime}\left(K^{ \pm}, K^{* \pm}\right)$ and $B^{ \pm} \rightarrow \eta\left(K^{ \pm}, K^{* \pm}\right)$. Expressing the charm quark content in the $\eta^{\prime}$ meson in terms of the matrix element $\left\langle\eta^{\prime}\left|\bar{c} \gamma_{\mu} \gamma_{5} c\right| 0\right\rangle=-i f_{\eta^{\prime}}^{(c)} q_{\mu}$, we find using data on the $J / \psi \rightarrow \eta_{c} \gamma$ and $J / \psi \rightarrow \eta^{\prime} \gamma$ decays that $\left|f_{\eta^{\prime}}^{(c)}\right| \simeq 5.8 \mathrm{MeV}$. The corresponding decay constant for $\eta$ meson is estimated to be $\left|f_{\eta}^{(c)}\right| \simeq 2.3 \mathrm{MeV}$ in the conventional $\left(\eta, \eta^{\prime}\right)$-mixing formalism and $\left|f_{\eta}^{(c)}\right| \simeq 0.93 \mathrm{MeV}$ in the $1 / N_{c}$-improved approach. The decrease in the value of $\left|f_{\eta}^{(c)}\right|$ reflects the small value of the 
singlet mixing angle $\theta_{0}$, which makes the $\eta$ an almost pure octet state [49], hence also reducing the $c \bar{c}$ component of the $\eta$-meson. Our estimate $\left|f_{\eta^{\prime}}^{(c)}\right| \simeq 5.8 \mathrm{MeV}$ is to be contrasted with the range $f_{\eta^{\prime}}^{(c)}=(50-180) \mathrm{MeV}$ obtained in [23]. (Likewise, we find $\left|f_{\eta^{\prime}}^{(c)} / f_{\eta^{\prime}}\right| \simeq 0.08$, which is also an order of magnitude smaller than the one given in [24].) We note that our estimate of $\left|f_{\eta}^{(c)}\right|$ is consistent with the bounds $-65 \mathrm{MeV} \leq f_{\eta}^{(c)} \leq 15 \mathrm{MeV}$, which have been obtained in the meanwhile from an analysis of the $Q^{2}$-dependence of the electromagnetic form factor of $\eta^{\prime}$.51]. Likewise, data on the electromagnetic form factor of $\eta$ is consistent with $\left|f_{\eta}^{(c)}\right|$ being small [51]. With our estimate of $\left|f_{\eta}^{(c)}\right|$, we find that this charm-induced contribution does not dominate the matrix element for $B^{ \pm} \rightarrow \eta^{\prime} K^{ \pm}$; the penguins play a more important role numerically in this decay.

The branching ratio $\mathcal{B}\left(B^{ \pm} \rightarrow \eta^{\prime} K^{ \pm}\right)$as well as those of the related ones $B^{ \pm} \rightarrow \eta^{\prime} K^{* \pm}$, $B^{ \pm} \rightarrow \eta K^{ \pm}$, and $B^{ \pm} \rightarrow \eta K^{* \pm}$, depend upon the interference of the amplitudes arising from the chain $b \rightarrow s(\bar{c} c) \rightarrow s\left(\eta^{\prime}, \eta\right)$, and the ones arising from calculating the matrix elements of the rest of the operators. Concentrating on the decay $B^{ \pm} \rightarrow \eta^{\prime} K^{ \pm}$, we note that the sign of the term involving the $b \rightarrow s(\bar{c} c) \rightarrow s\left(\eta^{\prime}, \eta\right)$ in the full amplitude is not determined a priori. Since the solutions with constructive or destructive interference terms are both logical possibilities, we have estimated $\mathcal{B}\left(B^{ \pm} \rightarrow \eta^{\prime} K^{ \pm}\right)$for both cases, with the positive- $f_{\eta^{\prime}}^{(c)}$ solution yielding marginally larger rate. However, more importantly, we find that the rate in this decay (and in some others) depends significantly on the parameter $\xi$. Hence, to make absolute predictions, the phenomenological value of this parameter has to be determined. We study a number of measured $B \rightarrow h_{1} h_{2}$ decays to estimate a range for $\xi$ which, given the present experimental errors and theoretical accuracy of our approach estimated by us as a factor 2 in rates, is understandably not very precise at this stage. The range $0 \leq \xi \leq 0.5$ is consistent with data.

This paper is organized as follows. In section 2, we review the effective Hamiltonian for the non-leptonic $B$ decays and calculate the matrix elements of the operators at the quark level in the NLL precision. In section 3, we formulate our factorization ansatz to calculate the hadronic matrix elements in the two-body decays $B \rightarrow h_{1} h_{2}$. The matrix elements for various decay modes of interest are also detailed here, together with a brief review of the mixing formalism for the $\eta-\eta^{\prime}-\eta_{c}$ sector. Our estimates of the decay constants $f_{\eta^{\prime}}^{(c)}$ and $f_{\eta}^{(c)}$ relevant for the decays $B^{ \pm} \rightarrow\left(\eta^{\prime}, \eta\right)\left(K^{ \pm}, K^{* \pm}\right)$ are also given here. Section 4 contains our numerical results. The input values for the various quantities (coupling constants, form factors, quark masses) are collected here in several tables. We compare the branching ratios with the CLEO data varying the factorization-related parameter $\xi$ and parameters of the CKM matrix [57]. The potential impact of some of these decays on the CKM phenomenology is illustrated in terms of the ratios of the branching ratios, which are more reliably calculable. In particular, the ratios $R_{1} \equiv \mathcal{B}\left(B^{0}\left(\overline{B^{0}}\right) \rightarrow \pi^{\mp} K^{ \pm}\right) / \mathcal{B}\left(B^{ \pm} \rightarrow \pi^{ \pm} K\right)$ and $R_{2} \equiv \mathcal{B}\left(B^{0}\left(\overline{B^{0}}\right) \rightarrow \pi^{\mp} h^{ \pm}\right) / \mathcal{B}\left(B^{ \pm} \rightarrow \pi^{ \pm} K\right)$ constrain the CKM-Wolfenstein parameter $\rho$ and $\eta$ [58]. The potential importance of $R_{1}$ in determining the angle $\gamma$ has been emphasized by Fleischer and Mannel [28, 29]. Interestingly, within the theoretical framework presented here, the measured ratio $R_{1}=0.65 \pm 0.40$ suggests (at $\pm 1 \sigma$ ) that $\rho \geq 0$, which in turn implies $\gamma \leq 90^{\circ}$, where $\gamma$ is one of the CP-violating angles of the unitarity triangle. We also comment on the effect of an (assumed) enhanced coefficient of the chromomagnetic operator, $C_{8}\left(m_{W}\right)$, in non-leptonic two-body $B$ decays. This scenario has been discussed in the context of new physics effects in $B$ decays [59, 60, 61]. We find, using the decay $B^{ \pm} \rightarrow K \pi^{ \pm}$, that varying the ratio $C_{8}\left(m_{W}\right) / C_{8}\left(m_{W}\right)^{S M}$ in a large range $( \pm 10)$ has no appreciable effect on the branching ratio within the present accuracy. Finally, we conclude 
with a summary in section 5 .

\section{Effective Hamiltonian for the non-leptonic decays $B \rightarrow$ $h_{1} h_{2}$}

We write the effective Hamiltonian $H_{\text {eff }}$ for the $\Delta B=1$ transitions as

$$
H_{e f f}=\frac{G_{F}}{\sqrt{2}}\left[V_{u b} V_{u q}^{*}\left(C_{1} O_{1}^{u}+C_{2} O_{2}^{u}\right)+V_{c b} V_{c q}^{*}\left(C_{1} O_{1}^{c}+C_{2} O_{2}^{c}\right)-V_{t b} V_{t q}^{*} \sum_{i=3}^{8} C_{i} O_{i}\right]
$$

where $q=d, s$ and $C_{i}$ are the Wilson coefficients evaluated at the renormalization scale $\mu$; the current-current operators $O_{1}^{u, c}$ and $O_{2}^{u, c}$ read

$$
\begin{array}{rlrl}
O_{1}^{u} & =\left(\bar{u}_{\alpha} b_{\alpha}\right)_{V-A}\left(\bar{q}_{\beta} u_{\beta}\right)_{V-A} & O_{1}^{c}=\left(\bar{c}_{\alpha} b_{\alpha}\right)_{V-A}\left(\bar{q}_{\beta} c_{\beta}\right)_{V-A} \\
O_{2}^{u}=\left(\bar{u}_{\beta} b_{\alpha}\right)_{V-A}\left(\bar{q}_{\alpha} u_{\beta}\right)_{V-A} & O_{2}^{c}=\left(\bar{c}_{\beta} b_{\alpha}\right)_{V-A}\left(\bar{q}_{\alpha} c_{\beta}\right)_{V-A}
\end{array}
$$

while the QCD penguin operators $\mathrm{O}_{3}-\mathrm{O}_{6}$ are

$$
\begin{array}{ll}
O_{3}=\left(\bar{q}_{\alpha} b_{\alpha}\right)_{V-A} \sum_{q^{\prime}}\left(\bar{q}_{\beta}^{\prime} q_{\beta}^{\prime}\right)_{V-A} & O_{5}=\left(\bar{q}_{\alpha} b_{\alpha}\right)_{V-A} \sum_{q^{\prime}}\left(\bar{q}_{\beta}^{\prime} q_{\beta}^{\prime}\right)_{V+A} \\
O_{4}=\left(\bar{q}_{\beta} b_{\alpha}\right)_{V-A} \sum_{q^{\prime}}\left(\bar{q}_{\alpha}^{\prime} q_{\beta}^{\prime}\right)_{V-A} & O_{6}=\left(\bar{q}_{\beta} b_{\alpha}\right)_{V-A} \sum_{q^{\prime}}\left(\bar{q}_{\alpha}^{\prime} q_{\beta}^{\prime}\right)_{V+A} .
\end{array}
$$

Finally, the dipole operators $O_{7}$ and $O_{8}$ read

$$
\begin{aligned}
& O_{7}=\left(e / 8 \pi^{2}\right) m_{b} \bar{s} \sigma^{\mu \nu}\left(1+\gamma_{5}\right) b F_{\mu \nu}, \\
& O_{8}=\left(g_{s} / 8 \pi^{2}\right) m_{b} \bar{s}_{\alpha} \sigma^{\mu \nu}\left(1+\gamma_{5}\right)\left(\lambda_{\alpha \beta}^{A} / 2\right) b_{\beta} G_{\mu \nu}^{A} .
\end{aligned}
$$

Here $\alpha$ and $\beta$ are the $S U(3)$ color indices and $\lambda_{\alpha \beta}^{A}, A=1, \ldots, 8$, are the Gell-Mann matrices. The subscripts $V \pm A$ represent the chiral projections $1 \pm \gamma_{5}$. Thus, in eqs. (2.2) and (2.3) $\left(\bar{u}_{\alpha} b_{\beta}\right)_{V-A}=\bar{u}_{\alpha} \gamma^{\mu}\left(1-\gamma_{5}\right) b_{\beta}$ etc. In eq. (2.4) $F_{\mu \nu}$ and $G_{\mu \nu}^{A}$ denote the photonic and the gluonic field strength tensor, respectively. We note that we neglect the effects of the operator $O_{7}$ in the present analysis as well as the so-called electro-weak penguin (4-Fermi) operators which we did not list explicitly. Likewise, the effect of weak annihilation and exchange diagrams will be neglected here. This is in line with the investigations reported in literature [19]. Working consistently to NLL precision, the coefficients $C_{1}-C_{6}$ are needed in NLL precision, while it is sufficient to use the LL value for $C_{8}$. The relevant Wilson coefficients to the desired accuracy are listed in table 1 for the two scales $\mu=5.0 \mathrm{GeV}$ and $\mu=2.5 \mathrm{GeV}$, where $C_{7}^{\text {eff }}=C_{7}-C_{5} / 3-C_{6}$ and $C_{8}^{e f f}=C_{8}+C_{5}$.

\subsection{Quark-level matrix elements}

The Wilson coefficients of the four-Fermi operators depend on the renormalization scale; in addition, in NLL precision, they also depend on the renormalization scheme. These unphysical dependences are compensated in principle by a corresponding scheme/scale dependence of the

matrix elements of the operators. However, when using the factorization ansatz for the hadronic 


\begin{tabular}{|c|r|r|}
\hline$C_{i}(\mu)$ & $\mu=5.0 \mathrm{GeV}$ & $\mu=2.5 \mathrm{GeV}$ \\
\hline \hline$C_{1}^{N L L}$ & 1.070 & 1.117 \\
$C_{2}^{N L L}$ & -0.166 & -0.257 \\
$C_{3}^{N L L}$ & 0.011 & 0.017 \\
$C_{4}^{N L L}$ & -0.031 & -0.044 \\
$C_{5}^{N N L}$ & 0.009 & 0.011 \\
$C_{6}^{N N L}$ & -0.037 & -0.056 \\
$C_{7}^{e f f, L L}$ & -0.303 & -0.338 \\
$C_{8}^{e f f, L L}$ & -0.144 & -0.158 \\
\hline
\end{tabular}

Table 1: Wilson coefficients $C_{i}(\mu)$ at the renormalization scale $\mu=5.0 \mathrm{GeV}$ and $\mu=2.5 \mathrm{GeV}$ in the NDR scheme. $C_{1}-C_{6}$ are in NLL accuracy, while $C_{7}^{e f f}$ and $C_{8}^{e f f}$ are in LL precision. For $\alpha_{s}(\mu)$ (in the $\overline{M S}$ scheme) we used the two-loop expression with 5 flavors and $\alpha_{s}^{\overline{M S}}\left(m_{Z}\right)=0.118$; $m_{t}^{\overline{M S}}\left(m_{t}\right)=165 \mathrm{GeV}$ (equivalent to $\left.m_{t, p o l e}=175 \mathrm{GeV}\right)$..

matrix elements of the operators, these cancellations do not take place in practice, because the factorized matrix elements of the operators are expressed in terms of decay constants and form factors, and are as such scheme/scale independent. To achieve this cancellation, we include perturbative QCD corrections to the partonic matrix element before doing the factorization step. We fully calculate the one-loop penguin-like diagrams in Fig. 11(a) and some process independent parts (see below) of the vertex correction diagrams associated with the four-Fermi operators, as shown in Figs. 2(a). These two classes of corrections are sufficient concerning the

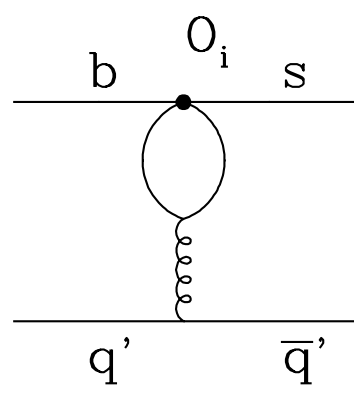

(a)

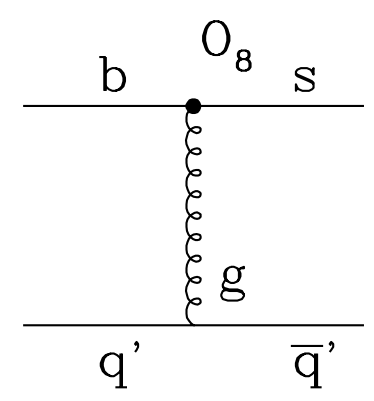

(b)

Figure 1: (a) Penguin-type diagrams associated with the four-Fermi operators $O_{i}(i=1, \ldots, 6)$; (b) Tree-level matrix element of the chromomagnetic dipole operator $O_{8}$.

cancellation of the scheme/scale dependences. Furthermore, the contribution associated with the operator $\mathrm{O}_{8}$, where the gluon splits into a quark - antiquark pair, as shown in Fig. 11(b) is of the same order in $\alpha_{s}$ as the corrections just mentioned and is therefore also taken into account in our analysis.

As we use in this paper the Wilson coefficients obtained in the naive dimensional scheme (NDR) with anti-commuting $\gamma_{5}$, we also have to evaluate the various $O\left(\alpha_{s}\right)$ corrections in this 


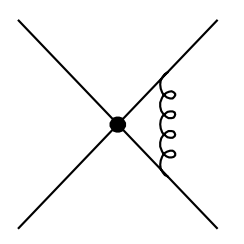

(a)

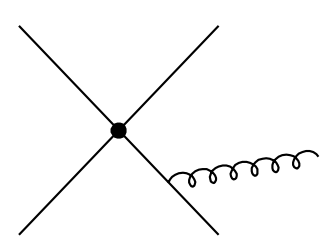

(b)

Figure 2: (a) Vertex correction diagrams to the four-Fermi operators $O_{i}(i=1, \ldots, 6)$;

(b) Corresponding Bremsstrahlung corrections.

scheme. These corrections can be absorbed into effective Wilson coefficients $C_{i}^{e f f}$, which for a general $S U(N)$ color group can be written as

$$
\begin{aligned}
C_{1}^{e f f} & =C_{1}+\frac{\alpha_{s}}{4 \pi}\left(r_{V}^{T}+\gamma_{V}^{T} \log \frac{m_{b}}{\mu}\right)_{1 j} C_{j}+\cdots \\
C_{2}^{e f f} & =C_{2}+\frac{\alpha_{s}}{4 \pi}\left(r_{V}^{T}+\gamma_{V}^{T} \log \frac{m_{b}}{\mu}\right)_{2 j} C_{j}+\cdots \\
C_{3}^{e f f} & =C_{3}-\frac{1}{2 N} \frac{\alpha_{s}}{4 \pi}\left(C_{t}+C_{p}+C_{g}\right)+\frac{\alpha_{s}}{4 \pi}\left(r_{V}^{T}+\gamma_{V}^{T} \log \frac{m_{b}}{\mu}\right)_{3 j} C_{j}+\cdots \\
C_{4}^{e f f} & =C_{4}+\frac{1}{2} \frac{\alpha_{s}}{4 \pi}\left(C_{t}+C_{p}+C_{g}\right)+\frac{\alpha_{s}}{4 \pi}\left(r_{V}^{T}+\gamma_{V}^{T} \log \frac{m_{b}}{\mu}\right)_{4 j} C_{j}+\cdots \\
C_{5}^{e f f} & =C_{5}-\frac{1}{2 N} \frac{\alpha_{s}}{4 \pi}\left(C_{t}+C_{p}+C_{g}\right)+\frac{\alpha_{s}}{4 \pi}\left(r_{V}^{T}+\gamma_{V}^{T} \log \frac{m_{b}}{\mu}\right)_{5 j} C_{j}+\cdots \\
C_{6}^{e f f} & =C_{6}+\frac{1}{2} \frac{\alpha_{s}}{4 \pi}\left(C_{t}+C_{p}+C_{g}\right)+\frac{\alpha_{s}}{4 \pi}\left(r_{V}^{T}+\gamma_{V}^{T} \log \frac{m_{b}}{\mu}\right)_{6 j} C_{j}+\cdots
\end{aligned}
$$

We have separated the contributions $C_{t}, C_{p}$, and $C_{g}$ arising from the penguin-type diagrams of the current-current operators $O_{1,2}$, the penguin-type diagrams of the operators $O_{3}-O_{6}$, and the tree-level diagram of the dipole operator $\mathrm{O}_{8}$, respectively. The process-independent contributions from the vertex-type diagrams are contained in the matrices $r_{V}$ and $\gamma_{V}$. Here $\gamma_{V}$ is that part of the anomalous matrix which is due to the vertex (and self-energy) corrections. This part can be easily extracted from $\hat{\gamma}^{(0)}$ in ref. [33]:

$$
\gamma_{V}=\left(\begin{array}{cccccc}
-2 & 6 & 0 & 0 & 0 & 0 \\
6 & -2 & 0 & 0 & 0 & 0 \\
0 & 0 & -2 & 6 & 0 & 0 \\
0 & 0 & 6 & -2 & 0 & 0 \\
0 & 0 & 0 & 0 & 2 & -6 \\
0 & 0 & 0 & 0 & 0 & -16
\end{array}\right)
$$

The matrix $r_{V}$ contains constant, i.e., momentum-independent parts associated with the vertex diagrams. This matrix can be extracted from the matrix $\hat{r}$ defined in eq. (2.12) (and given 
explicitly in eq. (4.6) in ref. [33]):

$$
r_{V}=\left(\begin{array}{cccccc}
\frac{7}{3} & -7 & 0 & 0 & 0 & 0 \\
-7 & \frac{7}{3} & 0 & 0 & 0 & 0 \\
0 & 0 & \frac{63}{27} & -\frac{63}{9} & 0 & 0 \\
0 & 0 & -7 & \frac{7}{3} & 0 & 0 \\
0 & 0 & 0 & 0 & -\frac{1}{3} & 1 \\
0 & 0 & 0 & 0 & -3 & \frac{35}{3}
\end{array}\right) .
$$

Note that the $\mu$ dependence and the scheme dependence of the vertex correction diagrams are fully taken into account in eq. (2.5) by the terms involving the matrices $\gamma_{V}$ and $r_{V}$, respectively. There are, however, still scheme-independent, process-specific terms omitted as indicated by the ellipses. When calculating inclusive quantities, such as the semileptonic branching ratios and $B$-hadron lifetimes, it is straightforward how to take these corrections into account. The virtual corrections are infrared divergent on their own, but together with the Bremsstrahlung contributions in Fig. 2(b), they lead to a finite and well defined $O\left(\alpha_{s}\right)$ correction, which is found to be small. However, it is less obvious how to include them in exclusive two-body decays. The point is that the division of the final states with and without the extra gluon is ambiguous and can be meaningfully defined only with a cut-off. As such a separation into virtual corrections and soft gluon Bremsstrahlung contributions is arbitrary anyhow, we only take into account the terms involving $\gamma_{V}$ and $r_{V}$. The explicit $O\left(\alpha_{s}\right)$ contributions which emerge from the penguin operators involving a $q \bar{q}$ pair in the loop are infrared finite on their own and hence do not require a cut-off.

The quantities $C_{t}, C_{p}$, and $C_{g}$ given by the diagrams shown in Fig. 11 read in the NDR scheme (after $\overline{M S}$ renormalization)

$$
\begin{gathered}
C_{t}=-C_{1}\left[\frac{V_{c b} V_{c q}^{*}}{V_{t b} V_{t q}^{*}} \tilde{C}_{t}\left(m_{c}\right)+\frac{V_{u b} V_{u q}^{*}}{V_{t b} V_{t q}^{*}} \tilde{C}_{t}\left(m_{u}\right)\right], \quad \tilde{C}_{t}(m)=\frac{2}{3}+\frac{2}{3} \log \frac{m^{2}}{\mu^{2}}-\Delta F_{1}\left(\frac{q^{2}}{m^{2}}\right), \\
C_{p}=C_{3}\left[\frac{4}{3}+\frac{2}{3} \log \frac{m_{q}^{2}}{\mu^{2}}+\frac{2}{3} \log \frac{m_{b}^{2}}{\mu^{2}}-\Delta F_{1}\left(\frac{q^{2}}{m_{q}^{2}}\right)-\Delta F_{1}\left(\frac{q^{2}}{m_{b}^{2}}\right)\right] \\
+\left(C_{4}+C_{6}\right) \sum_{i=u, d, s, c, b}\left[\frac{2}{3} \log \frac{m_{i}^{2}}{\mu^{2}}-\Delta F_{1}\left(\frac{q^{2}}{m_{i}^{2}}\right)\right] \\
C_{g}=-\frac{2 m_{b}}{\sqrt{\left\langle q^{2}\right\rangle}} C_{8}^{e f f}
\end{gathered}
$$

with $C_{8}^{e f f}=C_{8}+C_{5}$. The function $\Delta F_{1}(z)$ is defined as

$$
\Delta F_{1}(z)=-4 \int_{0}^{1} d x x(1-x) \log [1-z x(1-x)-i \epsilon]
$$

Two remarks are in order here. First, our expressions for $C_{i}^{e f f}$ in eq. (2.5) are written in terms of the Wilson coefficients in the NDR scheme. Analogous expressions (but with $r_{V}=0$, $\gamma_{V}=0$ and $C_{g}=0$ ) have been obtained earlier in the literature [10]. Comparing the expressions given here with the ones in [10], where the corresponding quantities $c_{3}^{\text {eff }}, \ldots, c_{6}^{\text {eff }}$ are expressed 
in terms of the so-called renormalization-scheme-independent Wilson coefficients $\bar{c}_{i}$ introduced in ref. [33], one notices that the constant terms appearing explicitly in $C_{t}$ and $C_{p}$ in the two papers are different. As the scheme dependence cancels automatically when including the oneloop matrix elements discussed above, we prefer to work with the Wilson coefficients in the NDR scheme.

Second, we have to explain the assumption which allows us to absorb the tree-level diagram $b \rightarrow s g \rightarrow s \bar{q}^{\prime} q^{\prime}$ associated with the operator $O_{8}$ into the contribution $C_{g}$ appearing in the expressions for $C_{i}^{e f f}$. It is straightforward to write down the matrix element

$$
\left\langle s \bar{q}^{\prime} q^{\prime}\left|O_{8}\right| b\right\rangle=-\frac{\alpha_{s}}{\pi} \frac{m_{b}}{q^{2}}\left(\bar{s}_{\alpha} \gamma_{\mu} q\left(1+\gamma_{5}\right) \frac{\lambda_{\alpha \beta}^{A}}{2} b_{\beta}\right)\left(\bar{q}_{\gamma}^{\prime} \gamma^{\mu} \frac{\lambda_{\gamma \delta}^{A}}{2} q_{\delta}^{\prime}\right),
$$

where $q$ is the momentum transferred by the gluon to the $\left(q^{\prime}, \bar{q}^{\prime}\right)$-pair. In the factorization model to be described below, $q^{\prime}$ and $\bar{q}^{\prime}$ cannot go into the same meson in the process $B \rightarrow h_{1} h_{2}$ due to color, i.e., $q^{\prime}$ goes into $h_{1}$, while $\bar{q}^{\prime}$ goes into $h_{2}$ or vice versa (see Fig. 1 (b)). The quantities $C_{t}, C_{p}$ and $C_{g}$ depend on the momentum $q$. Since we are interested here only in two-body decays, we assume for simplicity, that the three-momenta of $q^{\prime}$ and $\bar{q}^{\prime}$ are equal in magnitude but opposite in direction in the rest frame of the $b$-quark. The momentum transfer $q$ is then proportional to $p_{b}$, i.e.,

$$
q^{\mu}=\sqrt{\left\langle q^{2}\right\rangle} \frac{p_{b}^{\mu}}{m_{b}}
$$

where $\left\langle q^{2}\right\rangle$ is an averaged value of $q^{2}$. Inserting (2.13) into eq. (2.12) and using the equations of motion, the expression for $C_{g}$ in eq. (2.10) is readily obtained. To be consistent, we should also replace $q^{2}$ by $\left\langle q^{2}\right\rangle$ in the expressions for $C_{t}$ and $C_{p}$ in eqs. (2.8) and (2.9), respectively. To estimate the theoretical uncertainty introduced thereby, we treat $\left\langle q^{2}\right\rangle$ as a parameter which varies in the range $m_{b}^{2} / 4 \leq\left\langle q^{2}\right\rangle \leq m_{b}^{2} / 2$, following the prescriptions in literature [16, 17].

To summarize: The various $O\left(\alpha_{s}\right)$ corrections have been absorbed into effective Wilson coefficients $C_{i}^{e f f}(i=1, \ldots, 6)$; these coefficients are scheme independent and the term $\sim \alpha_{s} \log \mu$, which dominates the scale dependence of the original Wilson coefficients $C_{i}$ and the one-loop matrix elements, is absent in $C_{i}^{e f f}$. What remains to be done is to estimate the hadronic matrix elements $\left\langle h_{1} h_{2}\left|C_{i}^{e f f} O_{i}\right| B\right\rangle$ for $i=1, \ldots, 6$. The numerical values of the quantities $C_{i}^{e f f}$ are given in table 5 in section 4.1.4.

\section{Factorization ansatz for the matrix elements in $B \rightarrow$ $h_{1} h_{2}$}

We have now to work out the hadronic matrix elements of the operators $O_{i}(i=1, \ldots, 6)$ for the processes of interest. We use the factorization approximation, which we briefly explain for a specific example. Consider the matrix element due to the $u$-quark contribution of the operator $\mathrm{O}_{5}$ for the process $B^{-} \rightarrow K^{-} \omega$, i.e.,

$$
\left\langle K^{-} \omega\left|O_{5}^{(u)}\right| B^{-}\right\rangle \quad, \quad O_{5}^{(u)}=\left(\bar{s} \gamma_{\mu}\left(1-\gamma_{5}\right) b\right)\left(\bar{u} \gamma^{\mu}\left(1+\gamma_{5}\right) u\right)
$$

There are two contributing diagrams $D_{1}$ and $D_{2}$ shown in Fig. 3. The factorization approxi- 

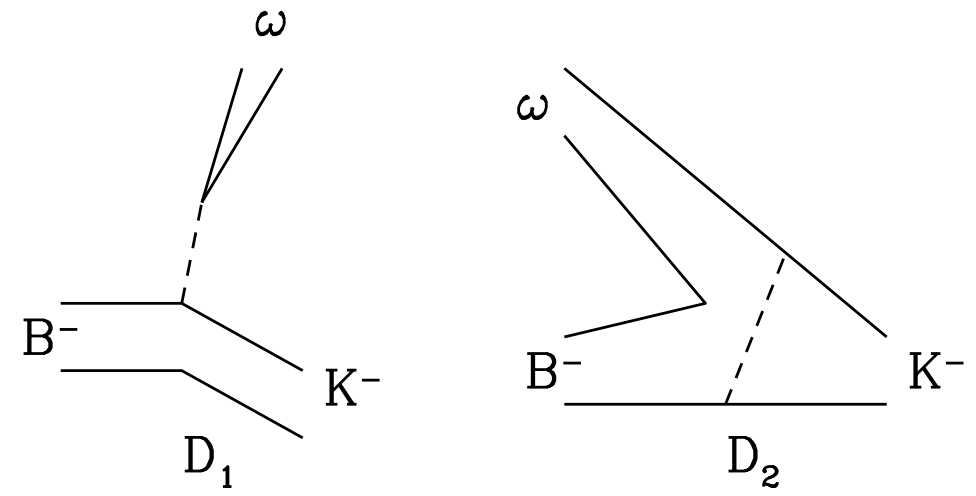

Figure 3: $D_{1}$ and $D_{2}$ are the two diagrams contributing in the factorization approximation. See text.

mation for $D_{1}$ is readily obtained:

$$
D_{1}=\left\langle\omega\left|\bar{u} \gamma^{\mu}\left(1+\gamma_{5}\right) u\right| 0\right\rangle\left\langle K^{-}\left|\bar{s} \gamma_{\mu}\left(1-\gamma_{5}\right) b\right| B^{-}\right\rangle=\left\langle\omega\left|\bar{u} u_{-}\right| 0\right\rangle\left\langle K^{-}\left|\bar{s} b_{-}\right| B^{-}\right\rangle
$$

where here and in the following the short-hand notation $\bar{q} q_{-}^{\prime}$ stands for

$$
\bar{q} q_{-}^{\prime}=\bar{q} \gamma_{\mu}\left(1-\gamma_{5}\right) q^{\prime}
$$

To get $D_{2}$ in the factorization approximation, we first write the operator $O_{5}^{(u)}$ in its Fierzed form

$$
\begin{aligned}
O_{5}^{(u)}= & -2\left(\bar{u}_{\beta}\left(1-\gamma_{5}\right) b_{\alpha}\right)\left(\bar{s}_{\alpha}\left(1+\gamma_{5}\right) u_{\beta}\right) \\
& =-2\left[\frac{1}{N}\left(\bar{u}\left(1-\gamma_{5}\right) b\right)\left(\bar{s}\left(1+\gamma_{5}\right) u\right)+\frac{1}{2}\left(\bar{u}\left(1-\gamma_{5}\right) \lambda b\right)\left(\bar{s}\left(1+\gamma_{5}\right) \lambda u\right)\right]
\end{aligned}
$$

where $\lambda$ denotes a color matrix. Only the first term in the square bracket in eq. (3.4) (being color singlet-singlet) contributes in the factorization approximation. One gets

$$
D_{2}=-\frac{2}{N}\left\langle\omega\left|\bar{u}\left(1-\gamma_{5}\right) b\right| B^{-}\right\rangle\left\langle K^{-}\left|\bar{s}\left(1+\gamma_{5}\right) u\right| 0\right\rangle
$$

using the Dirac equation, we can write $D_{2}$ as

$$
D_{2}=-\frac{2}{N} \frac{m_{K}^{2}}{\left(m_{s}+m_{u}\right)\left(m_{b}+m_{u}\right)}\left\langle\omega\left|\bar{u} b_{-}\right| B^{-}\right\rangle\left\langle K^{-}\left|\bar{s} u_{-}\right| 0\right\rangle .
$$

Doing analogous manipulations, the complete matrix element $M$ for $B^{-} \rightarrow \omega K^{-}$, defined as

$$
M=\left\langle\omega K^{-}\left|H_{e f f}\right| B^{-}\right\rangle \quad ; \quad H_{e f f}=\frac{G_{F}}{\sqrt{2}}\left[V_{u b} V_{u s}^{*}\left(C_{1}^{e f f} O_{1}^{u}+C_{2}^{e f f} O_{2}^{u}\right)-V_{t b} V_{t s}^{*} \sum_{i=3}^{6} C_{i}^{e f f} O_{i}\right],
$$


is then easily obtained. One gets

$$
\begin{aligned}
M= & \frac{G_{F}}{\sqrt{2}}\left\{V_{u b} V_{u s}^{*}\left(a_{1}\left\langle K^{-}\left|\bar{s} u_{-}\right| 0\right\rangle\left\langle\omega\left|\bar{u} b_{-}\right| B^{-}\right\rangle+a_{2}\left\langle K^{-}\left|\bar{s} b_{-}\right| B^{-}\right\rangle\left\langle\omega\left|\bar{u} u_{-}\right| 0\right\rangle\right)\right. \\
& -V_{t b} V_{t s}^{*}\left(\left(a_{4}-\frac{2 a_{6} m_{K}^{2}}{\left(m_{s}+m_{u}\right)\left(m_{b}+m_{u}\right)}\right)\left\langle K^{-}\left|\bar{s} u_{-}\right| 0\right\rangle\left\langle\omega\left|\bar{u} b_{-}\right| B^{-}\right\rangle\right. \\
& \left.\left.+2\left(a_{3}+a_{5}\right)\left\langle K^{-}\left|\bar{s} b_{-}\right| B^{-}\right\rangle\left\langle\omega\left|\bar{u} u_{-}\right| 0\right\rangle\right)\right\} .
\end{aligned}
$$

The quantities $a_{i}(i=1, \ldots, 6)$ are the following combinations of the effective Wilson coefficients in eq. (2.5):

$$
a_{2 i-1}=C_{2 i-1}^{e f f}+\frac{1}{N} C_{2 i}^{e f f} \quad, \quad a_{2 i}=C_{2 i}^{e f f}+\frac{1}{N} C_{2 i-1}^{e f f} \quad, \quad i=1,2,3 \quad .
$$

The explicit $1 / N$ terms in eq. (3.9) are always accompanied by an octet-octet contribution; this can be seen explicitly in eq. (3.4). As one discards this octet-octet contribution in the factorization approximation, one usually replaces $1 / N$ by $\xi$ and treats $\xi$ as a free parameter with the hope to compensate phenomenologically for the omitted octet-octet contribution in terms of a rescaled value of $\xi$. Note, however, that the $1 / N$ factors appearing explicitly in the (perturbative) expressions for the effective Wilson coefficients in eq. (2.5) are not replaced by $\xi$ in our work in contrast to [10], where also these $1 / N$ factors were replaced by $\xi$. We think that replacing also these $1 / N$ terms by $\xi$ destroys the scheme independence of the effective Wilson coefficients.

It is worth pointing out that the factorization Ansatz just discussed is the simplest one. Also, it is implicitly assumed that the relative strong phases (such as the ones arising from the final state interactions but also due to the non-perturbative contributions to the charmpenguins), contributing to the different $a_{i}$ 's are small. Of course, it does not mean that the strong interaction phases are assumed absent. The ones generated by the next-to-leading order perturbative QCD contributions from the charm penguins are taken into account. It remains to be seen if the non-perturbative phases from the competing tree and penguin contributions in the processes discussed here are indeed small.

Finally, before giving the matrix elements for the various exclusive two-body decays, we discuss the parametrization of the decay constants and form factors which appear in the factorized form of the hadronic matrix elements. The form factors are parametrized as

$$
\begin{aligned}
& \left\langle P\left(p^{\prime}\right)\left|V_{\mu}\right| B(p)\right\rangle=\left[\left(p+p^{\prime}\right)_{\mu}-\frac{m_{B}^{2}-m_{P}^{2}}{q^{2}} q_{\mu}\right] F_{1}\left(q^{2}\right)+\frac{m_{B}^{2}-m_{P}^{2}}{q^{2}} q_{\mu} F_{0}\left(q^{2}\right) \\
& \left\langle V\left(\epsilon, p^{\prime}\right)\left|\left(V_{\mu}-A_{\mu}\right)\right| B(p)\right\rangle=\frac{2}{m_{B}+m_{V}} i \epsilon_{\mu \nu \alpha \beta} \epsilon^{* \nu} p^{\alpha} p^{\prime \beta} V\left(q^{2}\right) \\
& -\left(m_{B}+m_{V}\right)\left[\epsilon_{\mu}^{*}-\frac{\epsilon^{*} \cdot q}{q^{2}} q_{\mu}\right] A_{1}\left(q^{2}\right)+ \\
& \frac{\epsilon^{*} \cdot q}{m_{B}+m_{V}}\left[\left(p+p^{\prime}\right)_{\mu}-\frac{m_{B}^{2}-m_{V}^{2}}{q^{2}} q_{\mu}\right] A_{2}\left(q^{2}\right)-\epsilon^{*} \cdot q \frac{2 m_{V}}{q^{2}} q_{\mu} A_{0}\left(q^{2}\right)
\end{aligned}
$$

where $P(V)$ is a pseudoscalar(vector) meson, $q=p-p^{\prime}$,

$$
A_{0}(0)=\frac{m_{B}+m_{V}}{2 m_{V}} A_{1}(0)-\frac{m_{B}-m_{V}}{2 m_{V}} A_{2}(0)
$$


and $F_{1}(0)=F_{0}(0)$. The decay constants $f_{P}$ and $f_{V}$ are defined as

$$
\left\langle 0\left|A_{\mu}\right| P(p)\right\rangle=i f_{P} p_{\mu} \quad, \quad\left\langle 0\left|V_{\mu}\right| V(\epsilon, p)\right\rangle=i f_{V} m_{V} \epsilon_{\mu} \quad .
$$

With these definitions we are in a position to write down the formulas for the matrix elements for the two body decays. They are given below explicitly for the four generic decay modes: $B \rightarrow \pi \pi, B \rightarrow K \pi, B^{ \pm} \rightarrow K^{ \pm} \omega, \pi^{ \pm} \omega$ and $B^{ \pm} \rightarrow\left(K^{ \pm}, K^{* \pm}\right)\left(\eta, \eta^{\prime}\right)$, which are also the ones we calculate numerically in the next section. However, the formalism given here is general and applicable to all two-body $B$-decays of the type $B \rightarrow P P, B \rightarrow P V$, and $B \rightarrow V V$.

\section{$3.1 \quad B \rightarrow \pi \pi$}

In this section we discuss the processes $B^{ \pm} \rightarrow \pi^{ \pm} \pi^{0}, B^{0}\left(\overline{B^{0}}\right) \rightarrow \pi^{ \pm} \pi^{\mp}$ and $B^{0}\left(\overline{B^{0}}\right) \rightarrow \pi^{0} \pi^{0}$.

\subsection{1 $\quad B^{ \pm} \rightarrow \pi^{ \pm} \pi^{0}$}

The matrix element $M$ for $B^{-} \rightarrow \pi^{-} \pi^{0}$ involves the operators $O_{1}^{u}$ and $O_{2}^{u}$ and reads (neglecting $\mathrm{SU}(2)$ breaking effects)

$$
M=\frac{G_{F}}{\sqrt{2}} V_{u b} V_{u d}^{*}\left(a_{1}+a_{2}\right)\left\langle\pi^{-}\left|\bar{d} u_{-}\right| 0\right\rangle\left\langle\pi^{0}\left|\bar{u} b_{-}\right| B^{-}\right\rangle
$$

with

$$
\left\langle\pi^{-}\left|\bar{d} u_{-}\right| 0\right\rangle\left\langle\pi^{0}\left|\bar{u} b_{-}\right| B^{-}\right\rangle=i f_{\pi}\left(m_{B}^{2}-m_{\pi}^{2}\right) F_{0}^{B \rightarrow \pi^{0}}\left(m_{\pi}^{2}\right)
$$

The branching ratio $\mathcal{B}\left(B^{-} \rightarrow \pi^{-} \pi^{0}\right)$ is then given by the expression

$$
\mathcal{B}\left(B^{-} \rightarrow \pi^{-} \pi^{0}\right)=\tau_{B} \frac{1}{8 \pi}|M|^{2} \frac{|p|}{m_{B}^{2}},
$$

where $\tau_{B}$ is the lifetime of the $B^{0}$-meson and $|p|$ is the absolute value of the 3-momentum of the $\pi^{-}$(or the $\pi^{0}$ ) in the rest frame of the $B^{0}$ meson. This expression for the branching ratio holds for other two-body decays being discussed with obvious changes of the indicated quantities. Hence, we shall give subsequently only the matrix elements $M$. Also, we shall give only the amplitudes for the decays of $B^{-}$and $\overline{B^{0}}$, and the matrix elements for the chargeconjugate processes are then obtained by complex conjugating the CKM factors. Since we are not addressing the question of CP-violation in this paper, all decay rates given later are to be interpreted in terms of the averaged branching ratios. Thus, for example, the branching ratio $\mathcal{B}\left(B^{ \pm} \rightarrow \pi^{ \pm} \pi^{0}\right)$ is defined as

$$
\mathcal{B}\left(B^{ \pm} \rightarrow \pi^{ \pm} \pi^{0}\right)=\frac{1}{2}\left(\mathcal{B}\left(B^{-} \rightarrow \pi^{-} \pi^{0}\right)+\mathcal{B}\left(B^{+} \rightarrow \pi^{+} \pi^{0}\right)\right)
$$

\subsection{2 $\quad B^{0}\left(\overline{B^{0}}\right) \rightarrow \pi^{+} \pi^{-}$}

The matrix element $M$ for $\overline{B^{0}} \rightarrow \pi^{-} \pi^{+}$reads

$$
\begin{aligned}
M= & \frac{G_{F}}{\sqrt{2}}\left[V_{u b} V_{u d}^{*} a_{1}-V_{t b} V_{t d}^{*}\left(a_{4}+\frac{2 a_{6} m_{\pi}^{2}}{\left(m_{b}-m_{u}\right)\left(m_{u}+m_{d}\right)}\right)\right] \times \\
& \left\langle\pi^{-}\left|\bar{d} u_{-}\right| 0\right\rangle\left\langle\pi^{+}\left|\bar{u} b_{-}\right| \bar{B}^{0}\right\rangle,
\end{aligned}
$$

with

$$
\left\langle\pi^{-}\left|\bar{d} u_{-}\right| 0\right\rangle\left\langle\pi^{+}\left|\bar{u} b_{-}\right| \bar{B}^{0}\right\rangle=i f_{\pi}\left(m_{B}^{2}-m_{\pi}^{2}\right) F_{0}^{B \rightarrow \pi^{-}}\left(m_{\pi}^{2}\right)
$$




\subsection{3 $\quad B^{0}\left(\overline{B^{0}}\right) \rightarrow \pi^{0} \pi^{0}$}

The matrix element $M$ for $\overline{B^{0}} \rightarrow \pi^{0} \pi^{0}$ reads

$$
\begin{aligned}
M= & \frac{G_{F}}{\sqrt{2}}\left[V_{u b} V_{u d}^{*} a_{2}+V_{t b} V_{t d}^{*}\left(a_{4}+\frac{a_{6} m_{\pi}^{2}}{m_{d}\left(m_{b}-m_{d}\right)}\right)\right] \times \\
& 2\left\langle\pi^{0}\left|\bar{u} u_{-}\right| 0\right\rangle\left\langle\pi^{0}\left|\bar{d} b_{-}\right| \bar{B}^{0}\right\rangle,
\end{aligned}
$$

with

$$
\left\langle\pi^{0}\left|\bar{u} u_{-}\right| 0\right\rangle\left\langle\pi^{0}\left|\bar{d} b_{-}\right| \bar{B}^{0}\right\rangle=i \frac{f_{\pi}}{\sqrt{2}}\left(m_{B}^{2}-m_{\pi}^{2}\right) F_{0}^{B \rightarrow \pi^{0}}\left(m_{\pi}^{2}\right) .
$$

When calculating the decay width, we have to take into account an extra factor $1 / 2$ due to the two identical particles in the final state.

\section{$3.2 \quad B \rightarrow K \pi, B \rightarrow K K$}

\subsection{1 $\quad B^{ \pm} \rightarrow K \pi^{ \pm}$}

The matrix element $M$ for $B^{-} \rightarrow \pi^{-} \bar{K}^{0}$ reads

$$
M=-\frac{G_{F}}{\sqrt{2}} V_{t b} V_{t s}^{*}\left[a_{4}+\frac{2 a_{6} m_{K}^{2}}{\left(m_{b}-m_{d}\right)\left(m_{s}+m_{d}\right)}\right]\left\langle\pi^{-}\left|\bar{d} b_{-}\right| B^{-}\right\rangle\left\langle\bar{K}^{0}\left|\bar{s} d_{-}\right| 0\right\rangle
$$

with

$$
\left\langle\pi^{-}\left|\bar{d} b_{-}\right| B^{-}\right\rangle\left\langle\bar{K}^{0}\left|\bar{s} d_{-}\right| 0\right\rangle=i f_{K}\left(m_{B}^{2}-m_{\pi}^{2}\right) F_{0}^{B \rightarrow \pi^{-}}\left(m_{K}^{2}\right) \quad .
$$

\subsection{2 $\quad B^{0}\left(\overline{B^{0}}\right) \rightarrow K^{ \pm} \pi^{\mp}$}

The matrix element $M$ for $\overline{B^{0}} \rightarrow \pi^{+} K^{-}$reads

$$
\begin{aligned}
M= & \frac{G_{F}}{\sqrt{2}}\left[V_{u b} V_{u s}^{*} a_{1}-V_{t b} V_{t s}^{*}\left(a_{4}+2 a_{6} \frac{m_{K}^{2}}{\left.\left(m_{b}-m_{u}\right)\left(m_{s}+m_{u}\right)\right)}\right)\right] \times \\
& \left\langle\pi^{+}\left|\bar{u} b_{-}\right| \bar{B}^{0}\right\rangle\left\langle K^{-}\left|\bar{s} u_{-}\right| 0\right\rangle
\end{aligned}
$$

with

$$
\left\langle\pi^{+}\left|\bar{u} b_{-}\right| \bar{B}^{0}\right\rangle\left\langle K^{-}\left|\bar{s} u_{-}\right| 0\right\rangle=i f_{K}\left(m_{B}^{2}-m_{\pi}^{2}\right) F_{0}^{B \rightarrow \pi^{-}}\left(m_{K}^{2}\right) \quad .
$$

3.2.3 $\quad B^{ \pm} \rightarrow K^{ \pm} \pi^{0}$

The matrix element $M$ for $B^{-} \rightarrow K^{-} \pi^{0}$ is given by

$$
\begin{aligned}
M= & \frac{G_{F}}{\sqrt{2}}\left[V_{u b} V_{u s}^{*}\left(a_{1}+a_{2} \frac{f_{\pi}}{\sqrt{2} f_{K}} \frac{m_{B}^{2}-m_{K}^{2}}{m_{B}^{2}-m_{\pi}^{2}} \frac{F_{0}^{B \rightarrow K^{-}}\left(m_{\pi}^{2}\right)}{F_{0}^{B \rightarrow \pi^{0}}\left(m_{K}^{2}\right)}\right)\right. \\
& \left.-V_{t b} V_{t s}^{*}\left(a_{4}+2 a_{6} \frac{m_{K}^{2}}{\left.\left(m_{b}-m_{u}\right)\left(m_{s}+m_{u}\right)\right)}\right)\right]\left\langle\pi^{0}\left|\bar{u} b_{-}\right| B^{-}\right\rangle\left\langle K^{-}\left|\bar{s} u_{-}\right| 0\right\rangle .
\end{aligned}
$$

with

$$
\left\langle K^{-}\left|\bar{s} u_{-}\right| 0\right\rangle\left\langle\pi^{0}\left|\bar{u} b_{-}\right| B^{-}\right\rangle=i f_{K}\left(m_{B}^{2}-m_{\pi}^{2}\right) F_{0}^{B \rightarrow \pi^{0}}\left(m_{K}^{2}\right)
$$




\subsection{4 $\quad B^{ \pm} \rightarrow K^{0} K^{ \pm}$}

The matrix element $M$ for $B^{-} \rightarrow K^{0} K^{-}$is given by

$$
M=-\frac{G_{F}}{\sqrt{2}} V_{t b} V_{t d}^{*}\left(a_{4}+2 a_{6} \frac{m_{K}^{2}}{\left.\left(m_{b}-m_{s}\right)\left(m_{s}+m_{d}\right)\right)}\right)\left\langle K^{-}\left|\bar{s} b_{-}\right| B^{-}\right\rangle\left\langle K^{0}\left|\bar{d} s_{-}\right| 0\right\rangle
$$

with

$$
\left\langle K^{0}\left|\bar{d} s_{-}\right| 0\right\rangle\left\langle K^{-}\left|\bar{s} b_{-}\right| B^{-}\right\rangle=i f_{K}\left(m_{B}^{2}-m_{K}^{2}\right) F_{0}^{B \rightarrow K}\left(m_{K}^{2}\right)
$$

3.3 $\quad B^{ \pm} \rightarrow K^{ \pm} \omega, \pi^{ \pm} \omega$

3.3.1 $\quad B^{ \pm} \rightarrow K^{ \pm} \omega$

The matrix element $M$ for $B^{-} \rightarrow K^{-} \omega$ reads

$$
\begin{aligned}
M= & \frac{G_{F}}{\sqrt{2}}\left\{V_{u b} V_{u s}^{*}\left[a_{1}+a_{2} \frac{F_{1}^{B \rightarrow K^{-}}\left(m_{\omega}^{2}\right)}{A_{0}^{B \rightarrow \omega}\left(m_{K}^{2}\right)} \frac{f_{\omega}}{\sqrt{2} f_{K}}\right]-V_{t b} V_{t s}^{*}\left[2\left(a_{3}+a_{5}\right) \frac{F_{1}^{B \rightarrow K^{-}}\left(m_{\omega}^{2}\right)}{A_{0}^{B \rightarrow \omega}\left(m_{K}^{2}\right)} \frac{f_{\omega}}{\sqrt{2} f_{K}}\right.\right. \\
& \left.\left.+a_{4}-\frac{2 a_{6} m_{K}^{2}}{\left(m_{b}+m_{u}\right)\left(m_{s}+m_{u}\right)}\right]\right\}\left\langle\omega\left|\bar{u} b_{-}\right| B^{-}\right\rangle\left\langle K^{-}\left|\bar{s} u_{-}\right| 0\right\rangle
\end{aligned}
$$

with

$$
\left\langle\omega\left|\bar{u} b_{-}\right| B^{-}\right\rangle\left\langle K^{-}\left|\bar{s} u_{-}\right| 0\right\rangle=-i f_{K} 2 m_{\omega}\left(p_{B} \cdot \epsilon_{\omega}^{*}\right) A_{0}^{B \rightarrow \omega}\left(m_{K}^{2}\right)
$$

There is only one non-vanishing helicity amplitude. In the rest frame of the decaying $B$ meson only longitudinally polarized $\omega$ 's are produced. $p_{B} \cdot \epsilon_{\omega}^{*}$ is then given by

$$
p_{B} \cdot \epsilon_{\omega}^{*}=\frac{m_{B}}{m_{\omega}}|p|
$$

where $|p|$ is the absolute value of the 3 -momentum of the $\omega$ (or the $K^{-}$) in the $B$ rest frame.

\subsection{2 $\quad B^{ \pm} \rightarrow \pi^{ \pm} \omega$}

The matrix element $M$ for $B^{-} \rightarrow \pi^{-} \omega$ reads

$$
\begin{aligned}
M= & \frac{G_{F}}{\sqrt{2}}\left\{V_{u b} V_{u d}^{*}\left[a_{1}+a_{2} \frac{F_{1}^{B \rightarrow \pi^{-}}\left(m_{\omega}^{2}\right)}{A_{0}^{B \rightarrow \omega}\left(m_{\pi}^{2}\right)} \frac{f_{\omega}}{\sqrt{2} f_{\pi}}\right]-V_{t b} V_{t d}^{*}\left[\left(2 a_{3}+a_{4}+2 a_{5}\right) \frac{F_{1}^{B \rightarrow \pi^{-}}\left(m_{\omega}^{2}\right)}{A_{0}^{B \rightarrow \omega}\left(m_{\pi}^{2}\right)} \frac{f_{\omega}}{\sqrt{2} f_{\pi}}\right.\right. \\
& \left.\left.+a_{4}-\frac{2 a_{6} m_{\pi}^{2}}{\left(m_{b}+m_{u}\right)\left(m_{d}+m_{u}\right)}\right]\right\}\left\langle\omega\left|\bar{u} b_{-}\right| B^{-}\right\rangle\left\langle\pi^{-}\left|\bar{d} u_{-}\right| 0\right\rangle
\end{aligned}
$$

with

$$
\left\langle\omega\left|\bar{u} b_{-}\right| B^{-}\right\rangle\left\langle\pi^{-}\left|\bar{d} u_{-}\right| 0\right\rangle=-i f_{\pi} 2 m_{\omega}\left(p_{B} \cdot \epsilon_{\omega}^{*}\right) A_{0}^{B \rightarrow \omega}\left(m_{\pi}^{2}\right) \quad .
$$

3.4 Mixing in the $\eta-\eta^{\prime}-\eta_{c}$ system and the decays $B^{ \pm} \rightarrow K^{ \pm} \eta^{\left({ }^{\prime}\right)}, K^{* \pm} \eta^{\left({ }^{\prime}\right)}$ Before we write the matrix elements for $B^{ \pm} \rightarrow K^{ \pm} \eta^{\prime}, B^{ \pm} \rightarrow K^{* \pm} \eta^{\prime}, B^{ \pm} \rightarrow K^{ \pm} \eta$ and $B^{ \pm} \rightarrow$ $K^{* \pm} \eta$ in the factorization approximation, we give a short discussion about the $\eta-\eta^{\prime}-\eta_{c}$ system. Our main interest for the decays mentioned above is to compute the hadronic matrix 
elements $\left\langle 0\left|\left(\bar{c} \gamma_{\mu} \gamma_{5}\right)\right| \eta\right\rangle$ and $\left\langle 0\left|\left(\bar{c} \gamma_{\mu} \gamma_{5}\right)\right| \eta^{\prime}\right\rangle$. The conventional $\left(\eta, \eta^{\prime}\right)$-mixing formalism involves a single mixing angle (called henceforth $\theta$ ) and it has been argued that it provides a satisfactory description of the decays involving $\eta$ and $\eta^{\prime}$ 47, 48]. However, recently the inadequacy of this mixing formalism has been pointed out in the context of the $1 / N_{c}$-improved chiral $U(3) \otimes U(3)$ perturbation theory [49, 50]. Instead, a formalism which involves two mixing angles in the $S U(3)$-octet and -singlet sector (called henceforth $\theta_{8}$ and $\theta_{0}$ ) is proposed. Since, the $S U(3)$ singlet component $\left|\eta_{0}\right\rangle$ in general mixes with the $|c \bar{c}\rangle$ component, introducing another angle (called $\theta_{c \bar{c}}$ ), we shall term the resulting mixing formalisms as the two-angle (involving $\theta$ and $\left.\theta_{c \bar{c}}\right)$ and three-angle (involving $\theta_{0}, \theta_{8}$ and $\theta_{c \bar{c}}$ ) frameworks.

\subsection{1 $\eta-\eta^{\prime}-\eta_{c}$ system in the two-angle mixing formalism}

Here, the physical $\eta$ and $\eta^{\prime}$ states are considered as mixtures of the $\eta_{8}$ and $\eta_{0}$ states 47 :

$$
|\eta\rangle=\cos \theta\left|\eta_{8}\right\rangle-\sin \theta\left|\eta_{0}\right\rangle \quad,\left|\eta^{\prime}\right\rangle=\sin \theta\left|\eta_{8}\right\rangle+\cos \theta\left|\eta_{0}\right\rangle
$$

where $\eta_{8}$ belongs to the $\mathrm{SU}(3)$ octet of the light pseudoscalar (Goldstone) mesons, while $\eta_{0}$ is an $\mathrm{SU}(3)$ singlet. In the quark basis they are given by

$$
\left|\eta_{8}\right\rangle=\frac{1}{\sqrt{6}}|u \bar{u}+d \bar{d}-2 s \bar{s}\rangle \quad,\left|\eta_{0}\right\rangle=\frac{1}{\sqrt{3}}|u \bar{u}+d \bar{d}+s \bar{s}\rangle
$$

The mixing angle $\theta$ can be extracted from the measured ratios 47]

$$
\frac{\Gamma(\eta \rightarrow \gamma \gamma)}{\Gamma\left(\pi^{0} \rightarrow \gamma \gamma\right)}=18\left(\frac{m_{\eta}}{m_{\pi}}\right)^{3} f_{\pi}^{2}\left[\frac{\cos \theta}{f_{8}} \frac{e_{u}^{2}+e_{d}^{2}-2 e_{s}^{2}}{\sqrt{6}}-\frac{\sin \theta}{f_{0}} \frac{e_{u}^{2}+e_{d}^{2}+e_{s}^{2}}{\sqrt{3}}\right]^{2}
$$

and

$$
\frac{\Gamma\left(\eta^{\prime} \rightarrow \gamma \gamma\right)}{\Gamma\left(\pi^{0} \rightarrow \gamma \gamma\right)}=18\left(\frac{m_{\eta^{\prime}}}{m_{\pi}}\right)^{3} f_{\pi}^{2}\left[\frac{\sin \theta}{f_{8}} \frac{e_{u}^{2}+e_{d}^{2}-2 e_{s}^{2}}{\sqrt{6}}+\frac{\cos \theta}{f_{0}} \frac{e_{u}^{2}+e_{d}^{2}+e_{s}^{2}}{\sqrt{3}}\right]^{2},
$$

where $e_{i}$ are the quark charges, $f_{\pi}, f_{8}$ and $f_{0}$ are the decay constants of the pion, the eighth component of the octet, and the singlet, respectively. Using $f_{8} / f_{\pi}=1.34 \pm 0.03$ [62 and the measured decay widths [46] $\Gamma\left(\pi^{0} \rightarrow \gamma \gamma\right)=(7.7 \pm 0.55) \mathrm{eV}, \Gamma(\eta \rightarrow \gamma \gamma)=(0.46 \pm 0.04) \mathrm{KeV}$, $\Gamma\left(\eta^{\prime} \rightarrow \gamma \gamma\right)=(4.26 \pm 0.19) \mathrm{KeV}$, one obtains

$$
\theta=-21.3^{o} \pm 2.5^{\circ} \quad ; \quad \frac{f_{0}}{f_{\pi}}=1.09 \pm 0.05
$$

It has been suggested in the context of the radiative decays $J / \psi \rightarrow \eta \gamma, \eta^{\prime} \gamma$ that they can be enacted by modelling them on the decay chain $J / \psi \rightarrow \eta_{c} \gamma \rightarrow\left(\eta, \eta^{\prime}\right) \gamma$, involving the Zweig-rule violating virtual transition $\eta_{c} \rightarrow \eta$ and $\eta_{c} \rightarrow \eta^{\prime}$. One can visualize these transitions taking

place via the two-gluon intermediate state. Since, only the $S U(3)$-singlet component of the $\eta$ and $\eta^{\prime}$ eigenstates is involved, one obtains a relation for the ratio $R_{J / \psi}\left(\eta / \eta^{\prime}\right)$ :

$$
R_{J / \psi}\left(\eta / \eta^{\prime}\right) \equiv \frac{\Gamma\left(J / \psi \rightarrow \eta^{\prime} \gamma\right)}{\Gamma(J / \psi \rightarrow \eta \gamma)}=\left(\frac{k_{\eta^{\prime}}}{k_{\eta}}\right)^{3} \frac{1}{\tan ^{2} \theta}
$$


where $k_{\eta^{\prime}}$ and $k_{\eta}$ denote the 3-momenta of $\eta^{\prime}$ and $\eta$, respectively. ¿From the measured ratio $\left(R_{J / \psi}\left(\eta / \eta^{\prime}\right)=5.0 \pm 0.8\right)$ 46], a value of $|\theta|=21.9^{\circ}$ can be extracted. Thus, one gets a consistent result from eqs. (3.39) and (3.40).

This Zweig-rule violating transition amplitude can also be formulated by postulating that the physical $\eta^{\prime}$ (as well as the $\eta$ ) has a small effective charm component, which should enable us to enact transitions of the type we are interested in, namely $b \rightarrow(c \bar{c}) s \rightarrow\left(\eta, \eta^{\prime}\right) s$. Thus, there is a contribution of the operators $O_{1,2}^{c}$ to the decay amplitude for the processes $B \rightarrow\left(K, K^{*}\right)\left(\eta^{\prime}, \eta\right)$, which can be modelled much the same way as the decays $J / \psi \rightarrow\left(\eta, \eta^{\prime}\right) \gamma$.

For $B^{ \pm} \rightarrow K^{ \pm} \eta^{\prime}$, for example, this yields in the factorization approximation

$$
M=-\frac{G_{F}}{\sqrt{2}} V_{c b} V_{c s}^{*} a_{2}\left\langle\eta^{\prime}(q)\left|\bar{c} \gamma_{\mu} \gamma_{5} c\right| 0\right\rangle\left\langle K\left(p^{\prime}\right)\left|\bar{s} \gamma^{\mu} b\right| B(p)\right\rangle
$$

The crucial quantity is the decay constant $f_{\eta^{\prime}}^{(c)}$ defined through the equation

$$
\left\langle\eta^{\prime}(q)\left|\bar{c} \gamma_{\mu} \gamma_{5} c\right| 0\right\rangle=-i f_{\eta^{\prime}}^{(c)} q_{\mu}
$$

The charm component comes in through the $\mathrm{SU}(3)$ singlet $\left|\eta_{0}\right\rangle$ which has a small charm admixture characterized by the mixing angle $\theta_{c \bar{c}}$ :

$$
\left|\eta_{0}\right\rangle=\frac{1}{\sqrt{3}}|u \bar{u}+d \bar{d}+s \bar{s}\rangle \cos \theta_{c \bar{c}}+|c \bar{c}\rangle \sin \theta_{c \bar{c}}
$$

The orthogonal state $\eta_{c}$ is then given by

$$
\left|\eta_{c}\right\rangle=-\frac{1}{\sqrt{3}}|u \bar{u}+d \bar{d}+s \bar{s}\rangle \sin \theta_{c \bar{c}}+|c \bar{c}\rangle \cos \theta_{c \bar{c}}
$$

Anticipating that the mixing angle $\theta_{c \bar{c}}$ is small, and dropping the $\sin ^{2} \theta_{c \bar{c}}$ term $\}$, eq. (3.43) reads approximately as

$$
\left|\eta_{0}\right\rangle=\frac{1}{\sqrt{3}}|u \bar{u}+d \bar{d}+s \bar{s}\rangle \cos \theta_{c \bar{c}}+\left|\eta_{c}\right\rangle \tan \theta_{c \bar{c}}
$$

$f_{\eta^{\prime}}^{(c)}$ and $f_{\eta_{c}}$ are then related through the equation

$$
f_{\eta^{\prime}}^{(c)}=\cos \theta \tan \theta_{c \bar{c}} f_{\eta_{c}}
$$

where $f_{\eta_{c}}$ is defined as $\left\langle\eta_{c}(p)\left|\bar{c} \gamma_{\mu} \gamma_{5} c\right| 0\right\rangle=-i f_{\eta_{c}} p_{\mu}$. We estimate the r.h.s. of eq. (3.46) using experimental data. First, the mixing angle $\theta_{c \bar{c}}$ can be extracted from the measured ratio

$$
R_{J / \psi}\left(\eta_{c} / \eta^{\prime}\right) \equiv \frac{\mathcal{B}\left(J / \psi \rightarrow \eta_{c} \gamma\right)}{\mathcal{B}\left(J / \psi \rightarrow \eta^{\prime} \gamma\right)}=\frac{(1.3 \pm 0.4) \times 10^{-2}}{(4.31 \pm 0.30) \times 10^{-3}} \simeq\left(\frac{k_{\eta_{c}}}{k_{\eta^{\prime}}}\right)^{3} \frac{1}{\cos ^{2} \theta \tan ^{2} \theta_{c \bar{c}}}
$$

which on using the central values of the measurements gives $\left|\theta_{c \bar{c}}\right|=0.014$. Second, the decay constant $f_{\eta_{c}}$ can be extracted from the measured decay width 46]

$$
\Gamma\left(\eta_{c} \rightarrow \gamma \gamma\right)=\frac{4(4 \pi \alpha)^{2} f_{\eta_{c}}^{2}}{81 \pi m_{\eta_{c}}}=7.5_{-1.4}^{+1.6} \mathrm{KeV}
$$

\footnotetext{
${ }^{1}$ If the mixing angle $\theta_{c \bar{c}}$ indeed turns out to be small, the extraction of the angle $\theta$ discussed above is not significantly altered.
} 
which, again for the central values, leads to $f_{\eta_{c}}=411 \mathrm{MeV}$. Eq. (3.48) is the result obtained using the standard nonrelativistic approach. This result also follows if one assumes that the shape of the distribution amplitudes for the charm and anti-charm quarks in the $\eta_{c}, \eta^{\prime}$ and $\eta$ mesons are very similar. This gives

$$
\left|f_{\eta^{\prime}}^{(c)}\right|=\left|\cos \theta \tan \theta_{c \bar{c}} f_{\eta_{c}}\right| \simeq 5.8 \mathrm{MeV}
$$

Similarly, we can estimate the charm content of the $\eta$ meson:

$$
\left\langle\eta(q)\left|\bar{c} \gamma_{\mu} \gamma_{5} c\right| 0\right\rangle=-i f_{\eta}^{(c)} q_{\mu}
$$

with

$$
\left|f_{\eta}^{(c)}\right|=\left|\sin \theta \tan \theta_{c \bar{c}} f_{\eta_{c}}\right| \simeq 2.3 \mathrm{MeV}
$$

Note that this method does not allow us to determine the signs of $f_{\eta^{\prime}}^{(c)}$ and $f_{\eta}^{(c)}$, because only the absolute value of the mixing angle $\theta_{c \bar{c}}$ can be extracted. To illustrate this ambiguity in the numerical results, we show in the case of $B^{ \pm} \rightarrow K^{ \pm} \eta^{\prime}$, the branching ratios for both signs.

\subsection{2 $\eta-\eta^{\prime}-\eta_{c}$ system in three-angle mixing formalism}

It has been recently argued that the octet-singlet mixing scheme involving $\left(\eta, \eta^{\prime}\right)$ described above needs modification [49, 50]. More specifically, one can show that taking into account $1 / N_{c}$ corrections in the effective $U(3) \otimes U(3)$ chiral perturbation theory, one needs to distinguish the mixing angles of the octet and singlet components. Restricting to the $\left(\eta, \eta^{\prime}\right)$ sector, one now has

$$
|\eta\rangle=\cos \theta_{8}\left|\eta_{8}\right\rangle-\sin \theta_{0}\left|\eta_{0}\right\rangle \quad,\left|\eta^{\prime}\right\rangle=\sin \theta_{8}\left|\eta_{8}\right\rangle+\cos \theta_{0}\left|\eta_{0}\right\rangle
$$

The analogous relations for the pseudoscalar decay constants and masses can be be derived from the terms quadratic in $\phi=\sum_{a=0, \ldots, 8} \lambda^{a} \phi_{a}$ in the chiral lagrangian for the pseudoscalar mesons $\phi_{a}$ (here, $\lambda^{a} ; a=1, \ldots, 8$ are the $S U(3)$ Gell-Mann matrices and $\lambda_{0}$ is a unit matrix). Writing the eigenstates as $\phi_{P}=\sum_{a} f_{P}^{a} \phi_{a}$, one can show that the vectors $\left(f_{\eta}^{8}, f_{\eta^{\prime}}^{8}\right)$ and $\left(f_{\eta}^{0}, f_{\eta^{\prime}}^{0}\right)$, defined through the matrix elements involving the axial-vector currents $A_{\mu}^{8}$ and $A_{\mu}^{0}$,

$$
\begin{aligned}
\left\langle 0\left|A_{\mu}^{8}\right| \eta(p)\right\rangle & =i f_{\eta}^{8} p_{\mu}, \\
\left\langle 0\left|A_{\mu}^{8}\right| \eta^{\prime}(p)\right\rangle & =i f_{\eta^{\prime}}^{8} p_{\mu}, \\
\left\langle 0\left|A_{\mu}^{0}\right| \eta(p)\right\rangle & =i f_{\eta}^{0} p_{\mu}, \\
\left\langle 0\left|A_{\mu}^{0}\right| \eta^{\prime}(p)\right\rangle & =i f_{\eta^{\prime}}^{0} p_{\mu}
\end{aligned}
$$

are not orthogonal to each other. Instead 49],

$$
\left(f_{\eta}^{8} f_{\eta}^{0}+f_{\eta^{\prime}}^{8} f_{\eta^{\prime}}^{0}\right)=-\frac{2 \sqrt{2}}{3}\left(f_{K}^{2}-f_{\pi}^{2}\right)(1+O(\delta))
$$

where $O(\delta)$ represents $O\left(1 / N_{c}\right)$ corrections. This relation then implies

$$
\sin \left(\theta_{0}-\theta_{8}\right)=\frac{2 \sqrt{2}}{3} \frac{\left(f_{K}^{2}-f_{\pi}^{2}\right)}{f_{8}^{2}}(1+O(\delta)),
$$


which yields (on using the decay widths $\Gamma(\eta \rightarrow 2 \gamma), \Gamma\left(\eta^{\prime} \rightarrow 2 \gamma\right)$ and the chiral perturbation theory result $\left.f_{8}=1.28 f_{\pi}\right)$ the following values [49]

$$
\theta_{8}=-20.5^{0}, \quad \theta_{0} \simeq-4.0^{0} .
$$

Thus, numerically the octet mixing angle $\theta_{8}$ comes out close to the angle $\theta$ but the singlet mixing angle is quite small. This implies that the pseudscalar meson $|\eta\rangle$ is almost a pure octet. Extending this formalism to the $\left(\eta, \eta^{\prime}, \eta_{c}\right)$ mixing, along the lines described in the previous subsection, now yields the following estimates of the quantities $f_{\eta^{\prime}}^{(c)}$ and $f_{\eta}^{(c)}$ :

$$
\begin{array}{r}
f_{\eta^{\prime}}^{(c)}=\cos \theta_{0} \tan \theta_{c \bar{c}} f_{\eta_{c}} \\
f_{\eta}^{(c)}=-\sin \theta_{0} \tan \theta_{c \bar{c}} f_{\eta_{c}} .
\end{array}
$$

Using again the ratio $R_{J / \psi}\left(\eta_{c} / \eta^{\prime}\right)$ given in eq. (3.47) yields $\left|f_{\eta^{\prime}}^{(c)}\right| \simeq 5.8 \mathrm{MeV}$ (the same as before), but $f_{\eta}^{(c)}=-f_{\eta^{\prime}}^{(c)} \tan \theta_{0}$, which is considerably smaller than the previous estimate of the same, as $\theta_{0} \ll \theta$.

The $\left(\eta, \eta^{\prime}\right)$-mixing framework with two angles $\theta_{0}$ and $\theta_{8}$ fares better than the conventional one from a phenomenological point of view as well. Feldmann and Kroll [51] have compared the two mixing frameworks in a recent analysis of the $\eta \gamma$ and $\eta^{\prime} \gamma$ transition form factors using data from CLEO [52], L3 [53], TPC [54] and CELLO [55], the decay widths $\Gamma(\eta \rightarrow 2 \gamma), \Gamma\left(\eta^{\prime} \rightarrow 2 \gamma\right)$ and the ratio $R_{J / \psi}\left(\eta / \eta^{\prime}\right)$. They show that even after allowing for a moderate $S U(3)$-breaking effect, one gets a poor fit of the data in the conventional mixing formalism (i.e., with the single angle $\theta$ ). However, the mentioned data can be well fit in the two-angle framework for the $\left(\eta, \eta^{\prime}\right)$-mixing. Their best-fit values yield (with $f_{\eta}^{(c)}$ and $f_{\eta^{\prime}}^{(c)}$ set to zero) [51]:

$$
\theta_{8}=-22.2^{0}, \quad \theta_{0}=-9.1^{0}, \quad \frac{f_{8}}{f_{\pi}}=1.28, \quad \frac{f_{0}}{f_{\pi}}=1.20
$$

which agrees reasonably well with the estimates of these parameters using chiral perturbation theory 49]:

$$
\theta_{8}=-20.5^{0}, \quad \theta_{0}=-4.0^{0}, \frac{f_{8}}{f_{\pi}}=1.28, \quad \frac{f_{0}}{f_{\pi}}=1.25
$$

If accurate high- $q^{2}$ data were available, one could determine the coupling constants $f_{\eta}^{(c)}$ and $f_{\eta^{\prime}}^{(c)}$ from the $\eta \gamma$ and $\eta^{\prime} \gamma$ transition form factors, respectively. While the value $f_{\eta}^{(c)}=0$ is consistent with the data, the anaysis in [51] yields the following range for $f_{\eta^{\prime}}^{(c)}$ :

$$
-65 \mathrm{MeV} \leq f_{\eta^{\prime}}^{(c)} \leq 15 \mathrm{MeV} .
$$

This determination is somewhat model-dependent as it depends on some parameters related to the charm wave-function. In the analysis reported in [51], the shape of the distribution amplitudes corresponding to the charm quark in the $\eta$ and $\eta^{\prime}$ are assumed to be the same as for $\eta_{c}$. It is satisfying that the value obtained by us $\left|f_{\eta^{\prime}}^{(c)}\right| \simeq 5.8 \mathrm{MeV}$ from $R_{J / \psi}\left(\eta_{c} / \eta^{\prime}\right)$ lies within the range given in eq. (3.60).

In what follows, we shall adhere to the $1 / N_{c}$-improved chiral perturbation theory description of the $\left(\eta, \eta^{\prime}\right)$-mixing. For numerical estimates, we use the best-fit values given in eq. (3.58) above. We now discuss the decays $B^{ \pm}\left(\eta, \eta^{\prime}\right)\left(K^{ \pm}, K^{* \pm}\right)$. 


\subsection{3 $\quad B^{ \pm} \rightarrow K^{ \pm} \eta^{\prime}$}

The matrix element $M$ for $B^{-} \rightarrow K^{-} \eta^{\prime}$ reads in the factorization approximation

$$
\begin{aligned}
M= & \frac{G_{F}}{\sqrt{2}}\left\{V_{u b} V_{u s}^{*}\left[a_{2}+a_{1} \frac{m_{B}^{2}-m_{\eta^{\prime}}^{2}}{m_{B}^{2}-m_{K}^{2}} \frac{F_{0}^{B \rightarrow \eta^{\prime}}\left(m_{K}^{2}\right)}{F_{0}^{B \rightarrow K^{-}}\left(m_{\eta^{\prime}}^{2}\right)} \frac{f_{K}}{f_{\eta^{\prime}}^{u}}\right]+V_{c b} V_{c s}^{*} a_{2} \frac{f_{\eta^{\prime}}^{(c)}}{f_{\eta^{\prime}}^{u}}\right. \\
& -V_{t b} V_{t s}^{*}\left[2 a_{3}-2 a_{5}+\left(a_{3}-a_{5}+a_{4}+\frac{a_{6} m_{\eta^{\prime}}^{2}}{m_{s}\left(m_{b}-m_{s}\right)}\right) \frac{f_{\eta^{\prime}}^{s}}{f_{\eta^{\prime}}^{u}}-\frac{a_{6} m_{\eta^{\prime}}^{2}}{m_{s}\left(m_{b}-m_{s}\right)}\right. \\
& \left.\left.+\left(a_{4}+\frac{2 a_{6} m_{K}^{2}}{\left(m_{s}+m_{u}\right)\left(m_{b}-m_{u}\right)}\right) \frac{m_{B}^{2}-m_{\eta^{\prime}}^{2}}{m_{B}^{2}-m_{K}^{2}} \frac{F_{0}^{B \rightarrow \eta^{\prime}}\left(m_{K}^{2}\right)}{F_{0}^{B \rightarrow K^{-}}\left(m_{\eta^{\prime}}^{2}\right)} \frac{f_{K}}{f_{\eta^{\prime}}^{u}}\right]\right\}\left\langle K^{-}\left|\bar{s} b_{-}\right| B^{-}\right\rangle\left\langle\eta^{\prime}\left|\bar{u} u_{-}\right| 0\right\rangle
\end{aligned}
$$

with

$$
\left\langle K^{-}\left|\bar{s} b_{-}\right| B^{-}\right\rangle\left\langle\eta^{\prime}\left|\bar{u} u_{-}\right| 0\right\rangle=i f_{\eta^{\prime}}^{u}\left(m_{B}^{2}-m_{K}^{2}\right) F_{0}^{B \rightarrow K^{-}}\left(m_{\eta^{\prime}}^{2}\right) \quad .
$$

The term proportional to $V_{c b} V_{c s}^{*}$ in eq. (3.61) is due to the charm content of the $\eta^{\prime}$ as discussed above. In eqs. (3.61) and (3.62) the decay constants $f_{\eta^{\prime}}^{u}$ and $f_{\eta^{\prime}}^{s}$, defined as

$$
\left\langle 0\left|\bar{u} \gamma_{\mu} \gamma_{5} u\right| \eta^{\prime}\right\rangle=i f_{\eta^{\prime}}^{u} p_{\mu} \quad, \quad\left\langle 0\left|\bar{s} \gamma_{\mu} \gamma_{5} s\right| \eta^{\prime}\right\rangle=i f_{\eta^{\prime}}^{s} p_{\mu}
$$

are given in terms of $f_{8}$ and $f_{0}$ as

$$
f_{\eta^{\prime}}^{u}=\frac{f_{8}}{\sqrt{6}} \sin \theta_{8}+\frac{f_{0}}{\sqrt{3}} \cos \theta_{0} \quad, \quad f_{\eta^{\prime}}^{s}=-2 \frac{f_{8}}{\sqrt{6}} \sin \theta_{8}+\frac{f_{0}}{\sqrt{3}} \cos \theta_{0} \quad .
$$

We remark that the matrix element $\left\langle 0\left|\bar{s} \gamma_{5} s\right| \eta^{\prime}\right\rangle$, which occurs when factorizing the contributions of $O_{5}$ and $O_{6}$, has to be treated with some care. In the earlier version of the this paper we erroneously used the relation

$$
\left\langle 0\left|\bar{s} \gamma_{5} s\right| \eta^{\prime}\right\rangle=-i \frac{f_{\eta^{\prime}}^{s} m_{\eta^{\prime}}^{2}}{2 m_{s}}
$$

which is vitiated due to the contribution of the anomaly term in the equation

$$
\partial^{\mu} \bar{s} \gamma_{\mu} \gamma_{5} s=2 m_{s} \bar{s} i \gamma_{5} s+\frac{\alpha_{s}}{4 \pi} G^{\alpha \beta} \tilde{G}_{\alpha \beta}
$$

To get the correct expression for the matrix element $\left\langle\eta^{\prime}\left|\bar{s} \gamma_{5} s\right| 0\right\rangle$, we now use instead the anomalyfree equation for the divergence of the octet axial-vector current,

$$
\partial^{\mu}\left(\bar{u} \gamma_{\mu} \gamma_{5} u+\bar{d} \gamma_{\mu} \gamma_{5} d-2 \bar{s} \gamma_{\mu} \gamma_{5} s\right)=2 i\left(m_{u} \bar{u} \gamma_{5} u+m_{d} \bar{d} \gamma_{5} d-2 m_{s} \bar{s} \gamma_{5} s\right)
$$

Neglecting the terms proportional to $m_{u}$ and $m_{d}$ on the r.h.s. of eq. (3.67) one derives

$$
\left\langle 0\left|\bar{s} \gamma_{5} s\right| \eta^{\prime}\right\rangle=i \frac{\sqrt{6} f_{8} \sin \theta_{8} m_{\eta^{\prime}}^{2}}{4 m_{s}}=-i \frac{\left(f_{\eta^{\prime}}^{s}-f_{\eta^{\prime}}^{u}\right) m_{\eta^{\prime}}^{2}}{2 m_{s}} .
$$

Of course, this relation can also be derived by working with the divergence of the (anomalous) singlet axial vector current. This gives rise to the term $-a_{6} m_{\eta^{\prime}}^{2} / m_{s}\left(m_{b}-m_{s}\right)$ in eq. (3.61). Likewise, the amplitudes of the other processes $B^{-} \rightarrow K^{*-} \eta^{\prime}, B^{-} \rightarrow K \eta$ and $B^{-} \rightarrow K^{*-} \eta$ published in the earlier version of this paper also get modified. The corrected amplitudes are given below. It appears that this (anomaly-related) error has permeated the recent literature [73] and should be corrected accordingly. 


\subsection{4 $\quad B^{ \pm} \rightarrow K^{* \pm} \eta^{\prime}$}

The matrix element $M$ for $B^{-} \rightarrow K^{*-} \eta^{\prime}$ reads in the factorization approximation

$$
\begin{aligned}
M= & \frac{G_{F}}{\sqrt{2}}\left\{V_{u b} V_{u s}^{*}\left[a_{2}+a_{1} \frac{F_{1}^{B \rightarrow \eta^{\prime}}\left(m_{K^{*}}^{2}\right)}{A_{0}^{B \rightarrow K^{*}}\left(m_{\eta^{\prime}}^{2}\right)} \frac{f_{K^{*}}}{f_{\eta^{\prime}}^{u}}\right]+V_{c b} V_{c s}^{*} a_{2} \frac{f_{\eta^{\prime}}^{(c)}}{f_{\eta^{\prime}}^{u}}\right. \\
& -V_{t b} V_{t s}^{*}\left[2 a_{3}-2 a_{5}+\left(a_{3}-a_{5}+a_{4}-\frac{a_{6} m_{\eta^{\prime}}^{2}}{m_{s}\left(m_{b}+m_{s}\right)}\right) \frac{f_{\eta^{\prime}}^{s}}{f_{\eta^{\prime}}^{u}}+\frac{a_{6} m_{\eta^{\prime}}^{2}}{m_{s}\left(m_{b}+m_{s}\right)}\right. \\
& \left.\left.+a_{4} \frac{F_{1}^{B \rightarrow \eta^{\prime}}\left(m_{K^{*}}^{2}\right)}{A_{0}^{B \rightarrow K^{*}}\left(m_{\eta^{\prime}}^{2}\right)} \frac{f_{K^{*}}}{f_{\eta^{\prime}}^{u}}\right]\right\}\left\langle K^{*-}\left|\bar{s} b_{-}\right| B^{-}\right\rangle\left\langle\eta^{\prime}\left|\bar{u} u_{-}\right| 0\right\rangle
\end{aligned}
$$

with

$$
\left\langle K^{*-}\left|\bar{s} b_{-}\right| B^{-}\right\rangle\left\langle\eta^{\prime}\left|\bar{u} u_{-}\right| 0\right\rangle=-i f_{\eta^{\prime}}^{u} 2 m_{K^{*}}\left(p_{B} \epsilon_{K^{*}}^{*}\right) A_{0}^{B \rightarrow K^{*}}\left(m_{\eta^{\prime}}^{2}\right)
$$

\subsection{5 $\quad B^{ \pm} \rightarrow K^{ \pm} \eta$}

The matrix element $M$ for $B^{-} \rightarrow K^{-} \eta$ reads in the factorization approximation

$$
\begin{aligned}
M= & \frac{G_{F}}{\sqrt{2}}\left\{V_{u b} V_{u s}^{*}\left[a_{2}+a_{1} \frac{m_{B}^{2}-m_{\eta}^{2}}{m_{B}^{2}-m_{K}^{2}} \frac{F_{0}^{B \rightarrow \eta}\left(m_{K}^{2}\right)}{F_{0}^{B \rightarrow K^{-}}\left(m_{\eta}^{2}\right)} \frac{f_{K}}{f_{\eta}^{u}}\right]+V_{c b} V_{c s}^{*} a_{2} \frac{f_{\eta}^{(c)}}{f_{\eta}^{u}}\right. \\
& -V_{t b} V_{t s}^{*}\left[2 a_{3}-2 a_{5}+\left(a_{3}-a_{5}+a_{4}+\frac{a_{6} m_{\eta}^{2}}{m_{s}\left(m_{b}-m_{s}\right)}\right) \frac{f_{\eta}^{s}}{f_{\eta}^{u}}-\frac{a_{6} m_{\eta}^{2}}{m_{s}\left(m_{b}-m_{s}\right)}\right. \\
& \left.\left.+\left(a_{4}+\frac{2 a_{6} m_{K}^{2}}{\left(m_{s}+m_{u}\right)\left(m_{b}-m_{u}\right)}\right) \frac{m_{B}^{2}-m_{\eta}^{2}}{m_{B}^{2}-m_{K}^{2}} \frac{F_{0}^{B \rightarrow \eta}\left(m_{K}^{2}\right)}{F_{0}^{B \rightarrow K^{-}}\left(m_{\eta}^{2}\right)} \frac{f_{K}}{f_{\eta}^{u}}\right]\right\}\left\langle K^{-}\left|\bar{s} b_{-}\right| B^{-}\right\rangle\left\langle\eta\left|\bar{u} u_{-}\right| 0\right\rangle
\end{aligned}
$$

where

$$
\left\langle K^{-}\left|\bar{s} b_{-}\right| B^{-}\right\rangle\left\langle\eta\left|\bar{u} u_{-}\right| 0\right\rangle=i f_{\eta}^{u}\left(m_{B}^{2}-m_{K}^{2}\right) F_{0}^{B \rightarrow K^{-}}\left(m_{\eta}^{2}\right)
$$

with

$$
f_{\eta}^{u}=\frac{f_{8}}{\sqrt{6}} \cos \theta_{8}-\frac{f_{0}}{\sqrt{3}} \sin \theta_{0} \quad, \quad f_{\eta}^{s}=-2 \frac{f_{8}}{\sqrt{6}} \cos \theta_{8}-\frac{f_{0}}{\sqrt{3}} \sin \theta_{0}
$$

\subsection{6 $\quad B^{ \pm} \rightarrow K^{* \pm} \eta$}

The matrix element $M$ for $B^{-} \rightarrow K^{*-} \eta$ reads in the factorization approximation

$$
\begin{aligned}
M= & \frac{G_{F}}{\sqrt{2}}\left\{V_{u b} V_{u s}^{*}\left[a_{2}+a_{1} \frac{F_{1}^{B \rightarrow \eta}\left(m_{K^{*}}^{2}\right)}{A_{0}^{B \rightarrow K^{*}}\left(m_{\eta}^{2}\right)} \frac{f_{K^{*}}}{f_{\eta}^{u}}\right]+V_{c b} V_{c s}^{*} a_{2} \frac{f_{\eta}^{(c)}}{f_{\eta}^{u}}\right. \\
& -V_{t b} V_{t s}^{*}\left[2 a_{3}-2 a_{5}+\left(a_{3}-a_{5}+a_{4}-\frac{a_{6} m_{\eta}^{2}}{m_{s}\left(m_{b}+m_{s}\right)}\right) \frac{f_{\eta}^{s}}{f_{\eta}^{u}}+\frac{a_{6} m_{\eta}^{2}}{m_{s}\left(m_{b}+m_{s}\right)}\right. \\
& \left.\left.+a_{4} \frac{F_{1}^{B \rightarrow \eta}\left(m_{K^{*}}^{2}\right)}{A_{0}^{B \rightarrow K^{*}}\left(m_{\eta}^{2}\right)} \frac{f_{K^{*}}}{f_{\eta}^{u}}\right]\right\}\left\langle K^{*-}\left|\bar{s} b_{-}\right| B^{-}\right\rangle\left\langle\eta\left|\bar{u} u_{-}\right| 0\right\rangle
\end{aligned}
$$


with

$$
\left\langle K^{*-}\left|\bar{s} b_{-}\right| B^{-}\right\rangle\left\langle\eta\left|\bar{u} u_{-}\right| 0\right\rangle=-i f_{\eta}^{u} 2 m_{K^{*}}\left(p_{B} \epsilon_{K^{*}}^{*}\right) A_{0}^{B \rightarrow K^{*}}\left(m_{\eta}^{2}\right)
$$

\section{Input Parameters, Numerical Results and Comparison with the CLEO Data}

\subsection{Input parameters}

The matrix elements for the decay $B \rightarrow h_{1} h_{2}$ derived in the preceding section depend on the effective coefficients $a_{1}, \ldots, a_{6}$, quark masses, various form factors, coupling constants and the CKM parameters. In turn, the coefficients $a_{i}$ and the quark masses depend on the renormalization scale $\mu$ and the QCD scale parameter $\Lambda_{\overline{\mathrm{MS}}}$. We have fixed $\Lambda_{\overline{\mathrm{MS}}}$ using $\alpha_{S}\left(M_{Z}\right)=0.118$, which is the central value of the present world average $\alpha_{S}\left(M_{Z}\right)=0.118 \pm 0.003$ [63]. The scale $\mu$ is varied between $\mu=m_{b}$ and $\mu=m_{b} / 2$, but due to the inclusion of the NLL expressions, the dependence of the decay rates on $\mu$ is small and hence not pursued any further. To be specific, we use $\mu=2.5 \mathrm{GeV}$ in the following. The CKM matrix will be expressed in terms of the Wolfenstein parameters [58], $A, \lambda, \rho$ and the phase $\eta$. Since the first two are well-determined with $A=0.81 \pm 0.06, \lambda=\sin \theta_{C}=0.2205 \pm 0.0018$, we fix them to their central values. The other two are correlated and are found to lie (at 95\% C.L.) in the range $0.25 \leq \eta \leq 0.52$ and $-0.25 \leq \rho \leq 0.35$ from the CKM unitarity fits [64]. We take four representative points in the allowed $(\rho, \eta)$ contour. Their values and the legends used in drawing the figures are as follows:

1. $\rho=0.05, \eta=0.36$, yielding $\sqrt{\rho^{2}+\eta^{2}}=0.36$ (drawn as a solid curve)

2. $\rho=0.30, \eta=0.42$, yielding $\sqrt{\rho^{2}+\eta^{2}}=0.51$ (drawn as a dashed curve)

3. $\rho=0, \eta=0.22$, yielding $\sqrt{\rho^{2}+\eta^{2}}=0.22$ (drawn as a dashed-dotted curve)

4. $\rho=-0.20, \eta=0.45$, yielding $\sqrt{\rho^{2}+\eta^{2}}=0.49$ (drawn as a dotted curve).

They correspond to the central values of the fits in [64], maximum allowed value of $\left|V_{u b} / V_{c b}\right|$ with positive $\rho$, minimum allowed value of $\left|V_{u b} / V_{c b}\right|$, and maximum allowed value of $\left|V_{u b} / V_{c b}\right|$ with negative $\rho$, respectively. The CKM parameters are also an output from the measured non-leptonic $B$ decays and we shall illustrate the potential interest in this kind of analysis using some of the ratios of the branching ratios as an exercise. The rest of the input quantities used in our estimates for the branching ratios are collected in several tables. We discuss now these input values.

\subsubsection{Effective coefficients in the factorization scheme}

With the electroweak penguins and the so-called $W$-annihilation/exchange diagrams neglected, the amplitudes for the various decays depend on six coefficients, $a_{i}$, defined in section 3. Eventually, one should determine each one of them (or particular combinations thereof) by analyzing the specific decay modes most sensitive to these coefficients. This way, one can measure the deviation in each one of them from their values in perturbation theory and determine if this deviation (due to non-perturbative effects) can be described in terms of a few universal parameters. Perhaps, it should be remarked here that an analysis of the heavy to heavy transitions in 
two-body $B$ decays can be reasonably well described in terms of one parameter, called $\zeta$ in 13 , whose value seems to be universal. Following this, we do the simplest thing here by assuming that a single parameter $\xi$, defined in the preceding section, can be used to compensate for neglecting the octet-octet terms in all matrix elements of the decays $B \rightarrow h_{1} h_{2}$. This is motivated by the fact that the energy release in these decays is comparable. It remains an open question if the parameter $\xi$ introduced here in the decays such as $B \rightarrow K \pi$ is close to the corresponding parameter $\zeta$, entering, for example, in the decay $B \rightarrow D \pi$. We show the dependence of the branching ratios in several decay modes in the range $0 \leq \xi \leq 1$, with $\xi=1 / 3$ being the naive factorization value, i.e., if one uses factorization and neglects the octet-octet contribution in the matrix elements.

\subsubsection{Decay coupling constants and form factors}

For the various decay constants occurring in the formulas in section 3 we use the numerical values shown in table 2 . The values for $f_{\omega}, f_{K}, f_{K^{*}}$ and $f_{\pi}$ coincide with the central values quoted

\begin{tabular}{|r|r|r|r|r|r|r|r|}
\hline$f_{\omega}$ & $f_{K}$ & $f_{K^{*}}$ & $f_{\pi}$ & $f_{0}$ & $f_{8}$ & $\left|f_{\eta^{\prime}}^{(c)}\right|$ & $\left|f_{\eta}^{(c)}\right|$ \\
\hline 195 & 160 & 214 & 131 & 157 & 168 & 5.8 & 0.93 \\
\hline
\end{tabular}

Table 2: Decay constants in MeV.

in [13] extracted from data on the electromagnetic decays of $\omega$ and $\tau$ decays, respectively 46 . The decay constants $f_{\eta^{\prime}}^{u}, f_{\eta^{\prime}}^{s}, f_{\eta}^{u}$ and $f_{\eta}^{s}$ defined in eqs. (3.64) and (3.73) are obtained from the values for $f_{0}$ and $f_{8}$ in table 2 and by using $\theta_{8}=-22.2^{\circ}$ for the $\left(\eta, \eta^{\prime}\right)$ mixing angle [47]. The errors on the coupling constants in the table are small (typically $(1-3) \%$ ), except on $f_{\eta^{\prime}}^{(c)}$ and $f_{\eta}^{(c)}$, for which present data allow a determination with an error of $\pm 15 \%$ (assuming the mixing formalism holds).

The decays being considered here, such as $B \rightarrow \pi K$, involve light hadrons in the final state. The rates require the knowledge of the various form factors at $q^{2}=m_{h}^{2}$, where $m_{h}$ denotes a light hadron mass. Since $q^{2}=m_{h}^{2}$ is rather close to the point $q^{2}=0$, and a simple pole model is mostly used to implement the $q^{2}$ dependence in the form factors, we shall neglect this $q^{2}$-dependence in the form factors and equate $F_{0,1}^{B \rightarrow h}\left(q^{2}=m_{h}^{2}\right)=F_{0,1}^{B \rightarrow h}\left(q^{2}=0\right)$. Explicit calculations bear this out and find that the variation in the stated range is indeed small [13, 38, 39. The values used for the form factors $F_{0,1}^{B \rightarrow h}\left(q^{2}=0\right)$ and $A_{0}^{B \rightarrow h}\left(q^{2}=0\right)$ in our rate estimates are listed in Table 3. They are taken from [1], which are reproduced in most other calculations (see, for example, Table 1 in [39]). Note also, that the $S U(3)$-breaking effects in the form factors are neglected. They are typically of $O(20) \%$ [38].

\begin{tabular}{|c|c|c|c|c|c|c|c|c|}
\hline$F_{0,1}^{B \rightarrow K^{-}}$ & $F_{0,1}^{B \rightarrow \pi^{-}}$ & $F_{0,1}^{B \rightarrow \pi^{0}}$ & & $F_{0,1}^{B \rightarrow \eta^{\prime}}$ & & $F_{0,1}^{B \rightarrow \eta}$ & $A_{0}^{B \rightarrow \omega}$ & $A_{0}^{B \rightarrow K^{*}}$ \\
\hline 0.33 & 0.33 & $\frac{0.33}{\sqrt{2}}$ & 0.33 & $\frac{\sin \theta_{8}}{\sqrt{6}}+\frac{\cos \theta_{0}}{\sqrt{3}}$ & 0.33 & $\frac{\cos \theta_{8}}{\sqrt{6}}-\frac{\sin \theta_{0}}{\sqrt{3}}$ & $\frac{0.28}{\sqrt{2}}$ & 0.28 \\
\hline
\end{tabular}

Table 3: Form factors at $q^{2}=0$. 


\subsubsection{Current and constituent quark masses}

The quark masses enter our analysis in two different ways. First, they occur in the amplitudes involving penguin loops. We treat the internal quark masses in these loops as constituent masses rather than current masses. For them we use the following (renormalization scale independent) values:

$$
m_{b}=4.88 \mathrm{GeV}, \quad m_{c}=1.5 \mathrm{GeV}, m_{s}=0.5 \mathrm{GeV}, m_{d}=m_{u}=0.2 \mathrm{GeV} .
$$

Variation in a reasonable range of these parameters does not change the numerical results of the branching ratios significantly. The value of $m_{b}$ above is fixed to be the current quark mass value $\overline{m_{b}}\left(\mu=m_{b} / 2\right)=4.88 \mathrm{GeV}$, given below. Second, the quark masses $m_{b}, m_{s}, m_{d}$ and $m_{u}$ also appear through the equations of motion when working out the (factorized) hadronic matrix elements. In this case, the quark masses should be interpreted as current masses. Using $\overline{m_{b}}\left(m_{b}\right)=4.45 \mathrm{GeV}$ 66 and

$$
\overline{m_{s}}(1 \mathrm{GeV})=150 \mathrm{MeV} \quad, \quad \overline{m_{d}}(1 \mathrm{GeV})=9.3 \mathrm{MeV} \quad, \quad \overline{m_{u}}(1 \mathrm{GeV})=5.1 \mathrm{MeV},
$$

from [62], the corresponding values at the renormalization scale $\mu=2.5 \mathrm{GeV}$ are given in table 4 , together with other input parameters needed for our analysis.

\begin{tabular}{|r|r|r|r|r|r|r|r|r|}
\hline$\overline{m_{b}}$ & $\overline{m_{s}}$ & $\overline{m_{d}}$ & $\overline{m_{u}}$ & $\alpha_{s}\left(m_{Z}\right)$ & $\tau_{B}$ & $\left\langle q^{2}\right\rangle$ & $\theta_{8}$ & $\theta_{0}$ \\
\hline $4.88 \mathrm{GeV}$ & $122 \mathrm{MeV}$ & $7.6 \mathrm{MeV}$ & $4.2 \mathrm{MeV}$ & 0.118 & $1.60 \mathrm{ps}$ & $m_{b}^{2} / 2$ & $-22.2^{o}$ & $-9.1^{0}$ \\
\hline
\end{tabular}

Table 4: Quark masses and other input parameters. The running masses are given at the renormalization scale $\mu=2.5 \mathrm{GeV}$.

\subsubsection{Numerical values for the effective Wilson coefficients $C_{i}^{e f f}$}

From eqs. 2.8) - (2.10) it follows that the effective Wilson coefficients $C_{i}^{e f f}$ defined in eq. (2.5) are in general complex numbers, which depend on quarks masses and on the CKM matrix elements. Taking the quark masses listed in eq. (4.1) and using the central values for the CKM parameters from the unitarity fits [64] (i.e. $\rho=0.05$ and $\eta=0.36$ ), the effective Wilson coefficients $C_{i}^{e f f}$ at the renormalization scale $\mu=2.5 \mathrm{GeV}$ are shown in table 5 . We remark that the (almost) identical values of these coefficients in the first two columns $(b \rightarrow s$ and $\bar{b} \rightarrow \bar{s}$ ) reflects that the imaginary parts of these effective Wilson coefficients are essentially generated by strong interactions. The numerically differing entries in the other two columns $(b \rightarrow d$ and $\bar{b} \rightarrow \bar{d}$ ) reflect that the weak (CP-violating) and strong interaction phases in these decays are comparable.

\subsection{Numerical results and comparison with CLEO data}

Having stated our theoretical framework and the input parameters, we now present our results for the various decays of interest listed in the previous section. A word of caution concerning the accuracy of the absolute decay rates calculated by us is in order. As just displayed, there are many parameters involved in describing exclusive non-leptonic decays and while the decay 


\begin{tabular}{|l|c|c|c|c|}
\hline & $b \rightarrow s$ & $b \rightarrow \bar{s}$ & $b \rightarrow d$ & $b \rightarrow d$ \\
\hline$C_{1}^{\text {eff }}$ & 1.160 & 1.160 & 1.160 & 1.160 \\
$C_{2}^{\text {eff }}$ & -0.334 & -0.334 & -0.334 & -0.334 \\
$C_{3}^{\text {eff }}$ & $0.021+0.004 i$ & $0.021+0.004 i$ & $0.020+0.002 i$ & $0.022+0.006 i$ \\
$C_{4}^{\text {eff }}$ & $-0.052-0.011 i$ & $-0.051-0.011 i$ & $-0.048-0.007 i$ & $-0.053-0.017 i$ \\
$C_{5}^{\text {eff }}$ & $0.016+0.004 i$ & $0.016+0.004 i$ & $0.015+0.002 i$ & $0.017+0.006 i$ \\
$C_{6}^{\text {eff }}$ & $-0.064-0.011 i$ & $-0.063-0.011 i$ & $-0.060-0.007 i$ & $-0.065-0.017 i$ \\
\hline
\end{tabular}

Table 5: Effective Wilson coefficients $C_{i}^{\text {eff }}$ at the renormalization scale $\mu=2.5 \mathrm{GeV}$ for the various $b \rightarrow q(\bar{b} \rightarrow \bar{q})$ transitions. See text and eq. (2.8).

rates do not depend sensitively on all of them, and many input parameters are already well known, it is obvious that the predicted branching ratios do depend sensitively on some for which there is no alternative at present to using model-dependent estimates. The particular quantities in question are the decay form factors. Some of these form factors enter in other processes which have been measured (such as in the semileptonic and radiative $B$ decays) and the estimates being used are found to reproduce the data quite well, yet some others are not yet constrained by data directly. So, the estimates given below for the absolute decay rates have to be taken with an accuracy which is not better than a factor 2 . The additional uncertainty due to the parameter $\xi$ can not be judged at this stage. That can only be ascertained in future, if this framework proves to be a reasonable way to analyze heavy to light transitions in $B$ decays.

However, within this framework, the ratios of the branching ratios are much more stable, as many of the theoretical uncertainties (such as in the form factors, various scales, and quark masses) cancel out to a large extent. In some cases, the dependence on the parameter $\xi$ also cancels, or it is very weak. Hence, the ratios are more reliable and the experimental information on these ratios can eventually be used meaningfully to draw inferences on the fundamental parameters, such as $\rho$ and $\eta$.

\subsubsection{Branching ratios for $B \rightarrow \pi \pi$ modes}

We shall show the branching ratios of interest as a function of the parameter $\xi$ for four different set of values of the CKM parameters. Wherever available, the present measurements of the branching ratios at the $\pm 1 \sigma$ level are also shown on these figures (thick solid lines). All experimental numbers are taken from [1, 2, 3], and in showing the experimental results, we have added the statistical and systematic errors in quadrature. We start by showing in Fig. \the branching ratio $\mathcal{B}\left(B^{ \pm} \rightarrow \pi^{0} \pi^{ \pm}\right)$. The decay rate for this mode is sensitive to both the variation in $\xi$ and the CKM parameters. This is obvious from the quadratic dependence of the decay rate on the quantity $\left|V_{u b}\right|$. Also, it depends on the combination $a_{1}+a_{2}$. Hence, a measurement of this decay rate will yield information on these quantities. In quoting a range, we shall take $0 \leq \xi \leq 0.5$ (which is suggested by the combined analysis of all the present CLEO data on $B \rightarrow h_{1} h_{2}$ decays which we show later). We estimate,

$$
\mathcal{B}\left(B^{ \pm} \rightarrow \pi^{0} \pi^{ \pm}\right) \simeq(0.1-1.4) \times 10^{-5}
$$


which is uncertain by over an order of magnitude. However, the lower range corresponds to the rather small value of the CKM-factor, $\left|V_{u b} / V_{c b}\right|=0.05$, and is therefore somewhat unlikely. For the central value $\left|V_{u b} / V_{c b}\right|=0.08$, we estimate

$$
\mathcal{B}\left(B^{ \pm} \rightarrow \pi^{0} \pi^{ \pm}\right)=(0.3-0.6) \times 10^{-5}
$$

The present experimental upper limit is (at 90\% C.L.)

$$
\mathcal{B}\left(B^{ \pm} \rightarrow \pi^{0} \pi^{ \pm}\right)<2.0 \times 10^{-5}
$$

In Fig. 5, we show the branching ratio $\mathcal{B}\left(B^{0}\left(\overline{B^{0}}\right) \rightarrow \pi^{\mp} \pi^{ \pm}\right)$. Again, this decay mode is sensitive to $\xi$ and the CKM parameters, although the resulting uncertainty is less in this case than in $\mathcal{B}\left(B^{ \pm} \rightarrow \pi^{0} \pi^{ \pm}\right)$. Comparison of the model calculations with the present upper limit (at $90 \%$ C.L.)

$$
\mathcal{B}\left(B^{0}\left(\overline{B^{0}}\right) \rightarrow \pi^{\mp} \pi^{ \pm}\right)<1.5 \times 10^{-5}
$$

shows that this decay mode is expected to lie within a factor $2-3$ of the present upper limit and hence should be measured soon. Already, the present upper limit on this mode disfavors some extreme values of the CKM parameters corresponding to $\left|V_{u b} / V_{c b}\right|$ close to or in excess of 0.11 .

In Fig. 6, we show the branching ratio $\mathcal{B}\left(B^{0}\left(\overline{B^{0}}\right) \rightarrow \pi^{0} \pi^{0}\right)$. This branching ratio is not very sensitive to $\xi$ in the region $0 \leq \xi \leq 0.5$ but rises sharply as $\xi \rightarrow 1$. All the curves lie however significantly below the present upper limit [46]:

$$
\mathcal{B}\left(B^{0}\left(\overline{B^{0}}\right) \rightarrow \pi^{0} \pi^{0}\right)<9.1 \times 10^{-6} .
$$

Restricting to $0 \leq \xi \leq 0.5$, our model calculation yields

$$
\mathcal{B}\left(B^{0}\left(\overline{B^{0}}\right) \rightarrow \pi^{0} \pi^{0}\right) \simeq(0.5-2.0) \times 10^{-6} .
$$

\subsubsection{Branching ratios for $B \rightarrow \pi K$ modes}

In Fig. 7, we show the branching ratio $\mathcal{B}\left(B^{ \pm} \rightarrow \pi^{ \pm} K\right)$. This is a good decay mode, in principle, to determine the parameter $\xi$, as there is no perceptible dependence of the rate on the CKM parameters. In the indicated range $0 \leq \xi \leq 1$, the branching ratio varies by slightly more than a factor 2. The experimental measurement is (at $\pm 3.2 \sigma)$ :

$$
\mathcal{B}\left(B^{ \pm} \rightarrow \pi^{ \pm} K\right)=\left(2.3_{-1.0-0.2}^{+1.1+0.2} \pm 0.2\right) \times 10^{-5}
$$

Our estimated branching ratio is in agreement with data, and there is a slight preference for smaller values of $\xi$, with $\xi>0.7$ somewhat disfavored. Since the CKM-parametric dependence is small, this decay mode is useful to show the effects of the QCD corrections. In Fig. 8, we show the branching ratio $\mathcal{B}\left(B^{ \pm} \rightarrow \pi^{ \pm} K\right)$ as a function of the scaled variable $\left\langle q^{2} / m_{b}^{2}\right\rangle$, in the range $0 \leq\left\langle q^{2} / m_{b}^{2}\right\rangle \leq 1$, calculated for $\xi=0$. The dashed line corresponds to the LL approximation, whereas the dotted and solid lines correspond to the truncated NLL approximation, and the complete NLL approximation as discussed in section 2, respectively. The dotted curve amounts to what has been used in the analysis of the decay modes $\left(B^{ \pm} \rightarrow \pi^{ \pm} K\right)$ in [28, 29]. The effect of the complete NLL corrections is numerically important, and they tend to decrease the branching ratio as compared to what one estimates by including the charm penguins alone. 
In Fig. 9, we show the branching ratio $\mathcal{B}\left(B^{0}\left(\overline{B^{0}}\right) \rightarrow \pi^{ \pm} K^{\mp}\right)$. Like its charged partner, $\mathcal{B}\left(B^{ \pm} \rightarrow \pi^{ \pm} K\right)$ discussed above, this decay mode is also sensitive to the parameter $\xi$, though in this case there is a perceptible dependence of the rate on the CKM parameters as well. The observed branching ratio (at $\pm 5.6 \sigma)$ :

$$
\mathcal{B}\left(B^{0}\left(\overline{B^{0}}\right) \rightarrow \pi^{ \pm} K^{\mp}\right)=\left(1.5_{-0.4-0.1}^{+0.5+0.1} \pm 0.1\right) \times 10^{-5}
$$

is quite comfortably accommodated by our estimates.

Comparing Figs. 9 and 7, one sees that the dependence of these decay rates on $\xi$ is very similar, and hence in the ratio of branching ratios it almost cancels out. Defining this ratio by $R_{1}$

$$
R_{1} \equiv \frac{\mathcal{B}\left(B^{0}\left(\overline{B^{0}}\right) \rightarrow \pi^{ \pm} K^{\mp}\right)}{\mathcal{B}\left(B^{ \pm} \rightarrow \pi^{ \pm} K\right)},
$$

we show $R_{1}$ as a function of the CKM parameter $\rho$ in Fig. 10 for two values of the CKM parameter $\eta=0.52$ (upper curve) and $\eta=0.25$ (lower curve). We note that $R_{1}$ is rather insensitive to $\eta$ but it does depend sensitively on $\rho$. Using the present CLEO measurement of $R_{1}($ at $\pm 1 \sigma)$

$$
R_{1}=0.65 \pm 0.40
$$

Fig. 10 suggests that negative values of $\rho$ are disfavored. This can also be converted as a statement on the CP-violating phase $\gamma$. Since the Wolfenstein parameter $\eta$ is positive as determined from the constraint on $\epsilon_{K}, \rho>0$ implies $\gamma<90^{\circ}$. We recall that the bounds on $\gamma$ obtained from the CKM unitarity fits yield symmetric constraints, centred around $\gamma=90^{\circ}$ (or $\rho=0$ ). However, it should be remarked that the lower bound on the ratio of the weak mass differences in the $B_{s}^{0}-\overline{B_{s}^{0}}$ and $B_{d}^{0} \overline{B_{d}^{0}}$ systems, $\Delta M_{s} / \Delta M_{d}$, which at present is posted as $\Delta M_{s} / \Delta M_{d}>20.4$ at $95 \%$ C.L. [67], now cuts away a good part of the negative- $\rho$ (equivalently $\gamma>90^{\circ}$ ) region. A recent analysis gives (at 95\% C.L.): $32^{0} \leq \gamma \leq 122^{0}$ [68, which is no longer symmetric around $\gamma=90^{\circ}$. On the other hand, the model-independent constraints on $\gamma$ from $R_{1}$, discussed by Fleischer and Mannel [28], are such that they force $\gamma$ to lie in the range $0^{0} \leq \gamma \leq \gamma^{\max }$ or $180^{\circ}-\gamma^{\max } \leq \gamma \leq 180^{\circ}$, depending on the sign of $\cos \delta$, where $\delta$ is the strong phase-shift difference between the tree and penguin amplitudes in the decay $B^{0}\left(\overline{B^{0}}\right) \rightarrow \pi^{ \pm} K^{\mp}$. Since this phase difference is calculated in our model, the preferred solution is the one in which $\gamma$ lies in the first quadrant, or $0^{0} \leq \gamma \leq \gamma^{\max }$. Unfortunately, with the present experimental errors, the $95 \%$ C.L. limit on $\rho$ from $R_{1}$ (or on $\gamma^{\max }$ ) does not allow one to draw more quantitative conclusions on the value of $\gamma$ than what one gets from the CKM fits 64, 68. This is expected to change with improved data on $R_{1}$, if the value of $R_{1}$ is found to be considerably less than 1. Our analysis, carried out in the factorization framework, underlines the sensitive dependence of $R_{1}$ on $\rho$, with $\rho \leq 0$ disfavored (at $\pm 1 \sigma$ ) by the CLEO data on $R_{1}$. The effect of the present lower bound on $\Delta M_{s} / \Delta M_{d}$ on $\rho$ is qualitatively similar to the one from the present measurement of $R_{1}$, namely both prefer $\rho \geq 0$. For an updated CKM fits, see also 65.

\subsubsection{Branching ratios for the $B \rightarrow h^{ \pm} \pi$ and $B \rightarrow h^{ \pm} K$ modes}

The decay modes $B^{ \pm} \rightarrow h^{ \pm} \pi^{0}, B^{0}\left(\overline{B^{0}}\right) \rightarrow h^{ \pm} \pi^{\mp}$ and $B^{ \pm} \rightarrow h^{ \pm} K^{0}$ have been measured with impressive precisions. We compare our model estimates with these measurements. In Fig. 11, 
we show the branching ratio $\mathcal{B}\left(B^{ \pm} \rightarrow h^{ \pm} \pi^{0}\right)$. The decay rate in this case is mildly dependent on $\xi$, but more importantly on the CKM parameters. The experimental measurement (at $\pm 5.5 \sigma$ ):

$$
\mathcal{B}\left(B^{ \pm} \rightarrow \pi^{0} h^{ \pm}\right)=\left(1.6_{-0.5-0.2}^{+0.6+0.3} \pm 0.1\right) \times 10^{-5}
$$

is reproduced well by our model.

In Fig. 12, we compare our model estimates with the CLEO measurements (at $\pm 7.8 \sigma)$ :

$$
\mathcal{B}\left(B^{0}\left(\overline{B^{0}}\right) \rightarrow h^{ \pm} \pi^{\mp}\right)=\left(2.2_{-0.5}^{+0.6} \pm 0.1\right) \times 10^{-5} .
$$

Agreement between our model and data is good. The two curves (dashed and dotted) which lie outside the $\pm 1 \sigma$ bands correspond to large values of the ratio $\left|V_{u b} / V_{c b}\right|$, namely $\left|V_{u b} / V_{c b}\right|=0.11$, which is also outside of the $\pm 1 \sigma$ bound from direct measurements of $\left|V_{u b} / V_{c b}\right|$. So, all of these different pieces of data are giving a consistent picture.

In Fig. 13, we show our estimates for the branching ratio for the mode $B^{ \pm} \rightarrow h^{ \pm} K^{0}$, which has been measured (at $4.4 \sigma$ )

$$
\mathcal{B}\left(B^{ \pm} \rightarrow h^{ \pm} K^{0}\right)=\left(2.4_{-1.0-0.2}^{+1.1+0.2} \pm 0.2\right) \times 10^{-5} .
$$

This branching ratio has a very similar dependence on $\xi$ as in the decay $B^{ \pm} \rightarrow \pi^{ \pm} K^{0}$ and likewise has little dependence on the CKM parameters. Model estimates are in agreement with data for $\xi \leq 0.7$.

As another example of a ratio of branching ratios, which is sensitive to the CKM parameters, we define the ratio $R_{2}$

$$
R_{2} \equiv \frac{\mathcal{B}\left(B^{0}\left(\overline{B^{0}}\right) \rightarrow h^{ \pm} \pi^{\mp}\right)}{\mathcal{B}\left(B^{ \pm} \rightarrow \pi^{ \pm} K^{0}\right)}
$$

which like $R_{1}$ is less dependent on the other input parameters, including $\xi$. Since $\mathcal{B}\left(B^{ \pm} \rightarrow\right.$ $\left.\pi^{ \pm} K^{0}\right)$ is insensitive to the CKM parameters, the ratio $R_{2}$ reflects the CKM dependence of $\mathcal{B}\left(B^{0}\left(\overline{B^{0}}\right) \rightarrow h^{ \pm} \pi^{\mp}\right)$. We plot the ratio $R_{2}$ in Fig. 14 as a function of the phase $\eta$, for three values of $\rho: \rho=0.05$ (dashed curve), $\rho=0.35$ (solid curve) and $\rho=-0.25$, which coincides with the case $\rho=0.35$. The present experimental value of $R_{2}$ (at $\pm 1 \sigma$ ) is

$$
R_{2}=0.96 \pm 0.57
$$

This shows that with the stated significance $R_{2}$ disfavors large values of $\eta$ in excess of $\eta \geq 0.5$.

\subsubsection{Branching ratios for the $B^{ \pm} \rightarrow \omega K^{ \pm}$and $B^{ \pm} \rightarrow \omega h^{ \pm}$modes}

Next, we study the decays $B^{ \pm} \rightarrow \omega K^{ \pm}$and $B^{ \pm} \rightarrow \omega h^{ \pm}(h=\pi, K)$, which have also been measured by the CLEO collaboration [1], with the former having a branching ratios (at $\pm 3.3 \sigma$ )

$$
\mathcal{B}\left(B^{ \pm} \rightarrow \omega K^{ \pm}\right)=\left(1.2_{-0.5}^{+0.7} \pm 0.2\right) \times 10^{-5},
$$

and the latter (at $\pm 6.0 \sigma)$

$$
\mathcal{B}\left(B^{ \pm} \rightarrow \omega h^{ \pm}\right)=\left(2.5_{-0.7}^{+0.8} \pm 0.5\right) \times 10^{-5}
$$

These measurements are compared with our model calculations in Figs. 15 and 16, respectively. Both of these decays have an interesting dependence on the variable $\xi$. Taken the data at face 
value $( \pm 1 \sigma)$, a value for $\xi$ in the range $0.15 \leq \xi \leq 0.5$ and $\xi \geq 0.85$ are somewhat disfavored by data in the decay $B^{ \pm} \rightarrow \omega K^{ \pm}$. Curiously, the estimated branching ratio $\mathcal{B}\left(B^{ \pm} \rightarrow \omega K^{ \pm}\right)$ has its lowest value in the range $\xi=0.3 \pm 0.1$, and in this range it fails to reproduce the data by almost $2 \sigma$. This observation and the present measurement of $\mathcal{B}\left(B^{ \pm} \rightarrow \pi^{ \pm} K\right)$ as well as $\mathcal{B}\left(B^{ \pm} \rightarrow h^{ \pm} K\right)$, which disfavor $\xi \geq 0.7$ then imply that the preferred value of $\xi$ in our model is either in the range $0 \leq \xi \leq 0.15$, or else $\xi \simeq 0.5$. In this range, however, the estimated branching ratio is somewhat lower than the experimental one in $B^{ \pm} \rightarrow \omega h^{ \pm}$, but not by a large amount. Due to the fact that the data being discussed are the first ones of their kind and the uncertainties related to the parameters of the present theoretical framework have not been exhaustively studied, one can not draw too strong conclusions on the value of the parameter $\xi$ from this decay.

\subsubsection{Branching ratios for the $B^{ \pm} \rightarrow\left(\eta, \eta^{\prime}\right)\left(K^{ \pm}, K^{* \pm}\right)$ modes}

Finally, we take up the decay $B^{ \pm} \rightarrow \eta^{\prime} K^{ \pm}$, which has attracted a lot of theoretical attention recently. Compared to the decays considered so far, this decay and the related ones $B^{ \pm} \rightarrow \eta K^{ \pm}$, $B^{ \pm} \rightarrow \eta^{\prime} K^{* \pm}$ and $B^{ \pm} \rightarrow \eta K^{* \pm}$ have an extra contribution from the decay chain $b \rightarrow s c \bar{c} \rightarrow$ $s\left(\eta, \eta^{\prime}\right)$.

In Fig. 17 we show the branching ratio $\mathcal{B}\left(B^{ \pm} \rightarrow \eta^{\prime} K^{ \pm}\right)$as a function of $\xi$, varying the CKM parameters as indicated in section 4.1. Since we are not able to determine the sign of the coupling constant $f_{\eta^{\prime}}^{(c)}$ due to the sign ambiguity in the determination of the angle $\theta_{c}$, we show the result for both $f_{\eta^{\prime}}^{(c)}= \pm 5.8 \mathrm{MeV}$. Note that the $\xi$-dependence of this branching ratio results in a factor 2 uncertainty varying $\xi$ in the range $0 \leq \xi \leq 0.5$ for the positive- $f_{\eta^{\prime}}^{c}$ solution; the branching ratio is less sensitive to $\xi$ for the negative- $f_{\eta^{\prime}}^{c}$ case. The positive- $f_{\eta^{\prime}}^{(c)}$ solution yields a marginally higher branching ratio. The CKM-parametric dependence of this branching ratio is not very marked. Within the present uncertainties in the input parameters, we get, at $\xi \simeq 0$

$$
\mathcal{B}\left(B^{ \pm} \rightarrow \eta^{\prime} K^{ \pm}\right) \simeq(3-4) \times 10^{-5},
$$

which at $\xi=0.5$ falls down to the range

$$
\mathcal{B}\left(B^{ \pm} \rightarrow \eta^{\prime} K^{ \pm}\right) \simeq(2-3) \times 10^{-5} .
$$

This is to be compared with the CLEO measurement (at $\pm 5.5 \sigma$ )

$$
\mathcal{B}\left(B^{ \pm} \rightarrow \eta^{\prime} K^{ \pm}\right)=\left(7.1_{-2.1}^{+2.5} \pm 0.9\right) \times 10^{-5} .
$$

Given the experimental and theoretical errors, the model estimates and data are clearly not incompatible though, with the values of the parameters used by us, our estimates are somewhat on the lower side. Since, apart from the form factors, this branching ratio is sensitive to the value of the $s$-quark mass, with the branching ratio increasing as $m_{s}$ is decreased from its default value $m_{s}(\mu=2.5 \mathrm{GeV})=122 \mathrm{MeV}$ used by us, the decay rate can be made to agree with the CLEO data by optimising these parameters in an overall fit. This is not warranted at this stage.

The branching ratios for the decays $B^{ \pm} \rightarrow \eta^{\prime} K^{* \pm}, B^{ \pm} \rightarrow \eta K^{ \pm}$and $B^{ \pm} \rightarrow \eta K^{* \pm}$ are shown in Figs. 18, 19, and 20, respectively, for the values $f_{\eta^{\prime}}^{(c)}=-5.8 \mathrm{MeV}$ and $f_{\eta}^{(c)}=-0.93 \mathrm{MeV}$. The reason for selecting the negative sign is to be traced to the observation that the contribution 
of the amplitude $b \rightarrow(c \bar{c}) s \rightarrow\left(\eta, \eta^{\prime}\right) s$ can also be calculated using QCD-anomaly which fixes the signs of these constants to be negative [69]. The estimated branching ratios satisfy the respective present experimental bounds on them [1]. For $0 \leq \xi \leq 0.5$, we predict

$$
\mathcal{B}\left(B^{ \pm} \rightarrow \eta^{\prime} K^{* \pm}\right) \simeq(0.3-0.9) \times 10^{-6}
$$

The decays $B^{ \pm} \rightarrow \eta\left(K^{ \pm}, K^{ \pm *}\right)$ on the other hand do not depend very sensitively on the sign of $f_{\eta}^{(c)}$. We estimate $(0 \leq \xi \leq 0.5)$

$$
\begin{aligned}
\mathcal{B}\left(B^{ \pm} \rightarrow \eta K^{ \pm}\right) & \simeq(1.0-2.8) \times 10^{-6} \\
\mathcal{B}\left(B^{ \pm} \rightarrow \eta K^{* \pm}\right) & \simeq(1.0-2.8) \times 10^{-6} .
\end{aligned}
$$

Finally, we remark that scenarios with a greatly enhanced strength of the dipole operator $O_{8}$ have been entertained in the literature [59, 60, 61, with the view of bringing the existing theoretical estimates of the semileptonic branching ratios and charm counting in $B$ decays in better rapport with data. A greatly enhanced value of $C_{8}\left(m_{W}\right)$ will influence the branching ratios in some selected non-leptonic $B$ decay channel as well. However, this effect is diluted due to the contributions from other Wilson coefficients, which are assumed to have their SM values. Also, as emphasized in [59, 60], and more recently in [61], the strong mixing of the operators

$\mathrm{O}_{2}$ and $\mathrm{O}_{8}$ would require a very large enhancement in $C_{8}\left(m_{W}\right) / C_{8}^{S M}\left(m_{W}\right)$, typically $O(10)$, to have a measurable influence in $B$ decays, calculated at the scale $\mu \simeq m_{b}$, due to the effects of the renormalization group. Qualitatively, this picture also holds in the analysis of the exclusive non-leptonic $B$ decays discussed by us. We show a typical case $B^{ \pm} \rightarrow K \pi^{ \pm}$in Fig. 21, where the branching ratio for this mode is plotted as a function of the variable $C_{8}\left(m_{W}\right) / C_{8}^{S M}\left(m_{W}\right)$. Despite the large range of this variable, we find that the influence of such a markedly enhanced $C_{8}\left(m_{W}\right)$ on non-leptonic $B$ decays is marginal. In future, we hope that these matters will be scrutinized much more minutely. We conclude that the non-leptonic $B$-decays considered here do not require large enhancements of $C_{8}\left(m_{W}\right)$, or of any other Wilson coefficient, as they are by and large compatible with data with their SM values.

\section{Summary}

In the first part of this paper we have presented a theoretical framework to study two-body decays of $B$ mesons with two light mesons in the final state. First, we took into account the complete NLL corrections at the partonic level, improving thereby previous calculations. In particular, we have also included the effects of the chromomagnetic penguin operator in nonleptonic $B$ decays. These NLL order corrections are numerically important in the exclusive decay rates. Second, to estimate the hadronic matrix elements we assumed factorization and gave a parametrization for the so-called heavy-to-light transitions. In its most economic version, this brings in a single phenomenological parameter, called $\xi$, which has to be determined by comparing the predictions of this model with data. We have taken a first look at the available CLEO data and estimated that our model is compatible within the present theoretical and experimental errors with data in the range $0 \leq \xi \leq 0.5$. With more precise data one should be able to test our model and see if within reasonable accuracy one obtains a universal value for this quantity in the heavy-to-light sector. Alternatively, with more precise data in several 
decay modes, we propose to extract the effective coefficients $a_{1}, \ldots, a_{6}$ directly to determine the extent of non-perturbative effects in each one of them.

In the second part of this paper we have applied this framework in the analysis of the exclusive two-body $B$ decays, in which QCD penguins play an important role. Some of these decays have been measured recently by the CLEO collaboration [1, 2, 3], with which we compared our model calculations; we have also predicted the branching ratios for some related decay modes which have not been measured yet. While the formalism provided here is generally applicable to study all $B \rightarrow P P, B \rightarrow P V$ and $B \rightarrow V V$ decays, we have restricted ourselves to discussing the four generic cases: $B \rightarrow \pi \pi, B \rightarrow K \pi, B^{ \pm} \rightarrow \omega h^{ \pm}$and $B^{ \pm} \rightarrow\left(\eta, \eta^{\prime}\right)\left(K^{ \pm}, K^{* \pm}\right)$.

In particular, we have studied at some length the last class, involving the decay $B^{ \pm} \rightarrow K^{ \pm} \eta^{\prime}$ and the related ones. As the $\eta^{\prime}$ and $\eta$ mesons are composed of $u, d$ and $s$ quarks, the corresponding decay rates are particularly sensitive to interference effects among the several competing amplitudes involving the current-current and the QCD-penguins operators, as was pointed out by Lipkin some time ago [6]. In addition, the operators $O_{1,2}^{c}$ which induce transitions of the form $b \rightarrow s(c \bar{c}) \rightarrow s\left(\eta, \eta^{\prime}\right)$ have to be included. Estimates of the latter require a trustworthy evaluation of the $c \bar{c}$ component in the wave-function of the $\eta^{\prime}$ and $\eta$ mesons. We have used the mixing formalism involving the $\left(\eta, \eta^{\prime}, \eta_{c}\right)$-complex and data to determine the $c \bar{c}$ contents of these mesons. We find that this charm-induced contribution does not dominate the amplitudes for the processes involving $\eta^{\prime}$; the decay rate is more sensitive to the penguin contributions. Our paper provides the complete amplitudes showing all these individual contributions. This can be used in future analyses of more precise data to determine the $c \bar{c}$ components in $\eta$ and $\eta^{\prime}$. The estimates presented here with $\left|f_{\eta^{\prime}}^{(c)}\right|=6 \mathrm{MeV}$ yield $\mathcal{B}\left(B^{ \pm} \rightarrow K^{ \pm} \eta^{\prime}\right)=(2-4) \times 10^{-5}$. This is somewhat lower than the central value of the present measurement but compatible with the value obtained by fluctuating down the experimental error by $1 \sigma$. However, a simple answer about the large measured value of $\mathcal{B}\left(B^{ \pm} \rightarrow \eta^{\prime} K^{ \pm}\right)$, a question frequently asked, in terms of a single dominating amplitude is not readily available, though the penguins and the singlet component of the $\eta^{\prime}$ are certainly at the back of the enhanced branching ratio for this decay. In our analysis, we find that the measured rate in the $\eta^{\prime} K^{ \pm}$mode is only marginally (say a factor 2) larger than our model estimates and given the theoretical errors there is nothing anomalous about it. We also expect that the data will evolve with time so as to reduce the present discrepancy.

We have made predictions to test this interference pattern in the related decays involving $\eta$ and $\eta^{\prime}$. The resulting decay rates, which also reflect the built-in angular momentum differences between the states $K^{*}\left(\eta, \eta^{\prime}\right)$ and $K\left(\eta, \eta^{\prime}\right)$, show a certain hierarchy among the branching ratios. While the other three may turn out comparable with each other (within a factor $2-3$ ), we predict:

$$
\mathcal{B}\left(B^{ \pm} \rightarrow \eta^{\prime} K^{ \pm}\right) \gg \mathcal{B}\left(B^{ \pm} \rightarrow \eta^{\prime} K^{* \pm}\right)
$$

The measurement of $\mathcal{B}\left(B^{ \pm} \rightarrow \eta^{\prime} K^{ \pm}\right)$being the largest measured so far is in line with our analysis based on the SM. However, in our SM-based framework it would be difficult to accommodate a much larger branching ratio $\mathcal{B}\left(B^{ \pm} \rightarrow \eta^{\prime} K^{* \pm}\right)$ vitiating this hierarchy.

The rates for the other decays presented in our analysis are also in reasonable agreement with data, within the presently allowed CKM-parameter space. Based on our estimates presented here, we expect the decay mode $B^{0}\left(\overline{B^{0}}\right) \rightarrow \pi^{\mp} \pi^{ \pm}$to be measured within a factor $2-3$ below the present upper limit. We point out interesting inferences which present data allows to draw on the consistency of the SM. In particular, the ratios $R_{1}$ and $R_{2}$ involving the $K \pi$ and $\pi \pi$ final 
states appear very promising. Present measurements on these ratios are tantalizingly close to providing independent information on the CKM-Wolfenstein parameters $\rho$ and $\eta$. Their impact on the CKM phenomenology will only be determined with more precise data, to which we look forward with animated interest. For the time being, the standard model rules OK - also in the non-leptonic $B$ decays!

\section{Acknowledgements:}

We are very grateful to Tom Browder, Jim Smith, Tomasz Skwarnicki and Frank Würthwein for helpful correspondence concerning the CLEO data. In particular, we thank Jim Smith for sharing his insight on several points discussed in this report and for asking us incisive questions related to the CLEO data and our analysis. Helpful discussions with Gustav Kramer, Hans Kühn, Heiri Leutwyler, Peter Minkowski, Hubert Simma and Daniel Wyler on various theoretical issues discussed here are also thankfully acknowledged. We also thank Nilendra Deshpande, Bhaskar Dutta, Sechul Oh, Guido Martinelli and Amarjit Soni for correspondence and discussions on the earlier version of this manuscript, and Thorsten Feldmann and Peter Kroll for sending us an advanced copy of their paper.

Note added in Proofs: Since the publication of this paper, several related papers have appeared in which some of the issues discussed here are also investigated [70, 71, 72, 73].

\section{References}

[1] B. Behrens (CLEO Collaboration), talk presented at the conference on B Physics and CP violation, Honolulu, Hawaii, March 1997.

[2] J. Smith (CLEO Collaboration), talk presented at the 1997 Aspen Winter Conference on Particle Physics, Aspen, Colorado, 1997.

[3] F. Würthwein (CLEO Collaboration), talk presented at Rencontres du Moriond, QCD and high energy hadronic interactions, Les Arcs, March 1997, hep-ph/9706010.

[4] M. Bauer and B. Stech, Phys. Lett. B152 (1985) 380;

M. Bauer, B. Stech and M. Wirbel, Z. Phys. C34 (1987) 103.

[5] A.J. Buras , J.M. Gérard and R. Rückl, Nucl. Phys. B268 (1986) 16.

[6] H.J. Lipkin, Phys. Lett. B254 (1991) 247.

[7] H.Y.Cheng, Phys. Lett. B335 (1994) 428;

J.M. Soares, Phys. Rev. D51 (1995) 3518.

[8] A. Deandrea et al., Phys. Lett. B318 (1993) 549; ibid. 320 (1994) 170.

[9] W.F. Palmer and B. Stech, Phys. Rev. D48 (1993) 4174. 
[10] G. Kramer, W.F. Palmer and H. Simma, Nucl. Phys. B428 (1994) 77; Z. Phys. C66 (1995) 429.

[11] D. Du and L. Guo, Z. Phys. C75 (1997) 9.

[12] M. Ciuchini et al., preprint CERN-TH/97-30 hep-ph/9703353.

[13] M. Neubert and B. Stech, preprint CERN-TH/97-99 [hep-ph 9705292], to appear in Heavy Flavors, Second Edition, ed. A.J. Buras and M. Lindner (World Scientific, Singapore).

[14] M. Bander, D. Silverman and A.. Soni, Phys. Rev. Lett. 43 (1979) 242.

[15] J.M. Gérarad and W.S. Hou, Phys. Rev. Lett. 62 (1989) 855; Phys. Rev. D43 (1991) 2909.

[16] N.G. Deshpande and J. Trampetic, Phys. Rev. D41 (1990) 2926.

[17] H. Simma and D. Wyler, Phys. Lett. B272 (1991) 395.

[18] G. Kramer and W.F. Palmer, Phys. Rev. D45 (1992) 132; Phys. Lett. B279 (1992) 181; Phys. Rev. D46 (1992) 2969.

[19] M. Gronau, O.F. Hernàndez, D. London and J.L. Rosner; Phys. Rev. D52 (1995) 6356; ibid. 6374.

[20] N.G. Deshpande and X.-G. He, Phys. Rev. Lett. 74 (1995) 26 [E.: ibid. p. 4099]; ibid. 75 (1995) 3064.

[21] R. Fleischer, Phys. Lett. B365 (1996) 399.

[22] M. Ciuchini et al., preprint CERN-TH/97-47 hep-ph/9704274.

[23] I. Halperin and A. Zhitnitsky, preprints hep-ph/9704412], hep-ph/9705251, and hepph/9706251.

[24] E.V. Shuryak and A.R. Zhitnitsky, preprint NI-97033-NQF hep-ph/9706316.

[25] F. Yuan and K.-T. Chao, Phys. Rev. D56 (1997) 2495.

[26] D. Atwood and A. Soni, Phys. Lett. B405 (1997) 150.

[27] W.-S. Hou and B. Tseng, preprint hep-ph/9705304.

[28] R. Fleischer and T. Mannel. preprint TTP-97-17 hep-ph/9704423.

[29] R. Fleischer and T. Mannel. preprint TTP-97-22 [hep-ph/9706261.

[30] K.G. Wilson, Phys. Rev. 179 (1969) 1499.

[31] G. Altarelli and L. Maiani, Phys. Lett. B52 (1974) 351;

M.K. Gaillard and B.W. Lee, Phys. Rev. Lett. 33 (1974) 108;

G. Altarelli, G. Curci, G. Martinelli and S. Petrarca, Phys. Lett. B99 (1981) 141; Nucl. Phys. B187 (1981) 461;

A.J. Buras and P.H. Weisz, Nucl. Phys. B333 (1990) 66. 
[32] K. Chetyrkin, M. Misiak and M. Münz, Phys. Lett. B400 (1997) 206..

[33] A.J. Buras et al., Nucl. Phys. B370 (1992) 69.

[34] R.P. Feynman in Symmetries in Particle Physics, ed. A. Zichichi (Acad. Press, 1965) 167. O. Haan and B. Stech, Nucl. Phys. B22 (1970) 448.

[35] J. Ellis, M.K. Gaillard and D.V. Nanopoulos, Nucl. Phys. B100 (1975) 313;

D. Fakirov and B. Stech, Nucl. Phys. B133 (1978) 315.

[36] B. Grinstein, D. Scora, B. Grinstein and M.B. Wise, Phys. Rev. D39 (1989) 799.

[37] R. Casalbuoni et al., Phys. Lett. B292 (1992) 299; B299 (1993) 139.

[38] A. Ali, V.M. Braun and H. Simma, Phys. Rev. C63 (1994) 437.

[39] P. Ball and V.M. Braun, Phys. Rev. D55 (1997) 5561.

[40] D. Ebert, R.N. Faustov and V.O. Galkin, preprint DESY 97-102, HUB-EP-96/97.

[41] T.E. Browder, K.Honscheid and D. Pedrini, Ann. Rev. Nucl. Part. Sci. 46 (1996) 395.

[42] H. Georgi, Phys. Lett. B240 (1990) 447;

A. Falk, H. Georgi, B. Grinstein and M.B. Wise, Nucl. Phys. B343 (1990) 1.

[43] J.D. Bjorken in New Developments in High-Energy Physics, ed. E.G. Floratos and A. Verganelakis, Nucl. Phys. (Proc. Suppl.) 11 (1989) 321.

[44] Karl Berkelman (unpublished CLEO note) has emphasized the impact of $c \bar{c}$ content of $\eta^{\prime}$ and its implications in $B$ decays. We thank Jim Smith for bringing this to our attention.

[45] Jim Smith has also estimated the $c \bar{c}$ content in the $\eta^{\prime}$ meson using Gaussian wave functions for the hadrons (private communication).

[46] R.M. Barnett et al. (Particle Data Group), Phys. Rev. D54 (1996) 1.

[47] F.J. Gilman and R. Kaufman, Phys. Rev. D36 (1987) 2761. See also, Dynamics of the Standard Model, authors: J.F. Donoghue, E. Golowich and B.R. Holstein, Cambridge University Press, 1992.

[48] E.P. Venugopal and B.R. Holstein, preprint hep-ph/9710382.

[49] H. Leutwyler, preprint hep-ph/9709408.

[50] P. Herrera-Sikoldy, J.I. Latorre, P. Pascual and J. Taron, preprint hep-ph/9710268.

[51] T. Feldmann and P. Kroll, preprint WUB 97-28.

[52] J. Gronberg et al. (CLEO Collaboration), preprint hep-ex/9707031.

[53] M. Acciari et al. (L3 Collaboration), preprint PPE/97-110.

[54] H. Aihara et al. (TPC/2 $\gamma$ Collaboration), Phys. Rev. Lett. 64 (1990) 172. 
[55] H.J. Behrend et al. (CELLO Collaboration), Z. Phys. C49 (1991) 401.

[56] C. Greub, T. Hurth and D. Wyler, Phys. Lett. 380 (1996) 385; Phys. Rev. D54 (1996) 3350 .

[57] N. Cabibbo, Phys. Rev. Lett. 10 (1963) 531;

M. Kobayashi and K. Maskawa, Prog. Theor. Phys. 49 (1973) 652.

[58] L. Wolfenstein, Phys. Rev. Lett. 51 (1983) 1945.

[59] A.L. Kagan, Phys. Rev. D51 (1995) 6196;

A.L. Kagan and J. Rathman, preprint hep-ph/9701300.

[60] M. Ciuchini, E. Gabrielli and G.F. Giudice, Phys. Lett. B388 (1996) 353 [E. B393 (1997) 489].

[61] A. Lenz, U. Nierste and G. Ostermaier, preprint DESY 97-119 [hep-ph/9706501.

[62] J. Gasser and H. Leutwyler, Nucl. Phys. B250 (1985) 465.

[63] M. Schmelling, preprint hep-ex/9701002.

[64] A. Ali and D. London, Nucl. Phys. B (Proc. Suppl.) 54A (1997) 297;

A. Ali, Acta Physica Polonica B27 (1996) 3529.

[65] P. Paganini, E. Parodi, P. Roudeau and A. Stocchi, preprint hep-ph/9711261.

[66] M. Gremm, A. Kapustin, Z. Ligeti and M.B. Wise, Phys. Rev. Lett. 77 (1996) 20.

[67] H.G. Moser (ALEPH Collaboration), invited talk at the IV International Workshop on Progress in Heavy Quark Physics, Rostock, Sept. 20-22, 1997.

[68] A. Ali and D. London, in Proceedings of the IV International Workshop on Progress in Heavy Quark Physics, Rostock, Sept. 20-22, 1997.

[69] A. Ali, J. Chay, C. Greub and P. Ko (to be published).

[70] A. Datta, X.-G. He and S. Pakvasa, preprint UH-511-864-97, ISU-HET-97-07, hepph/9707259.

[71] A.S. Dighe, M. Gronau and J. Rosner, preprint hep-ph/9707521.

[72] H.Y. Cheng and B. Tseng, preprints hep-ph/9707316; hep-ph/9708211.

[73] N.G. Deshpande, B. Dutta and Sechul Oh, preprint hep-ph/9710354. 


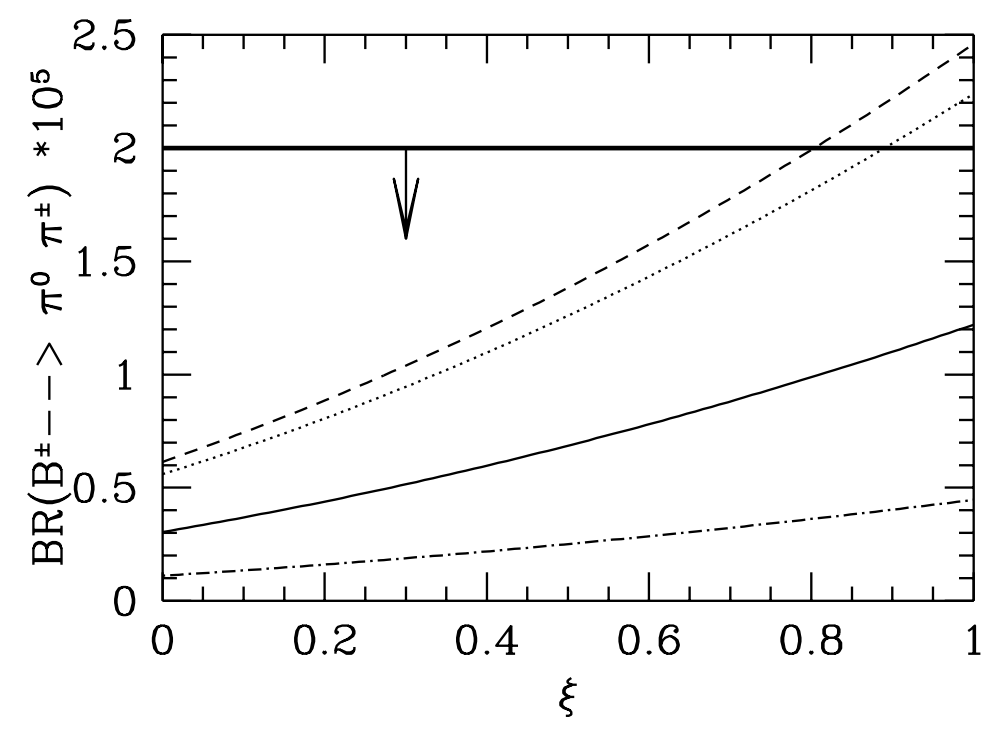

Figure 4: Branching ratio for $B^{ \pm} \rightarrow \pi^{0} \pi^{ \pm}$as a function of $\xi$ for various points in the $(\rho ; \eta)$ plane. Solid curve: $(\rho, \eta)=(0.05,0.36)$; dotted curve: $(\rho, \eta)=(-0.20,0.45)$; dashed curve: $(\rho, \eta)=(0.30,0.42)$; dashed-dotted curve: $(\rho, \eta)=(0.00,0.22)$. The horizontal thick solid line (with the arrow) shows the CLEO upper limit (at 90\% C.L.).

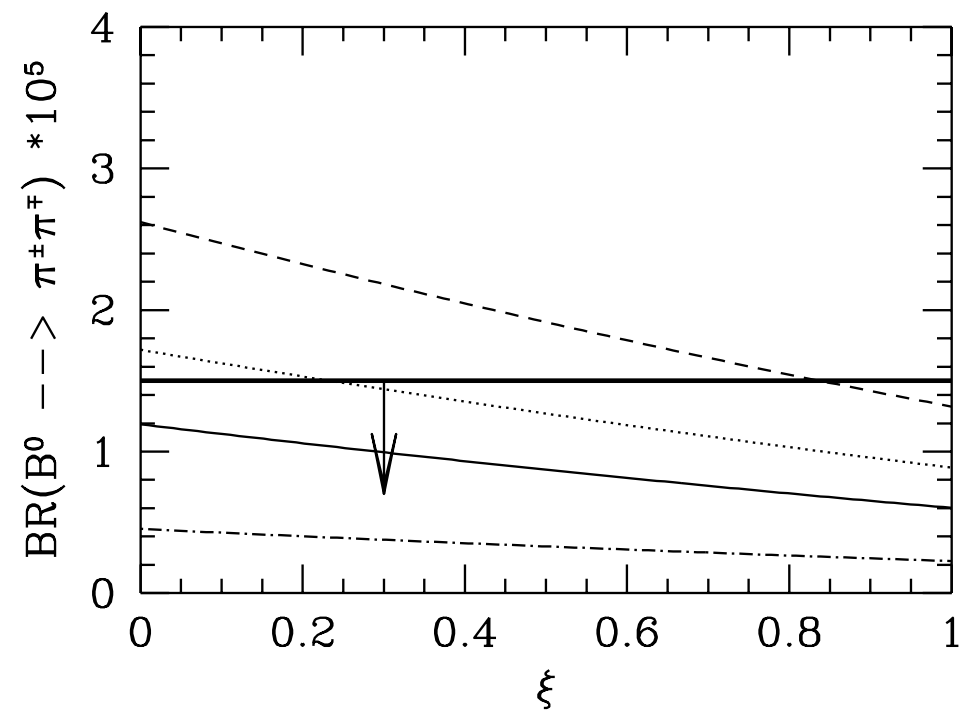

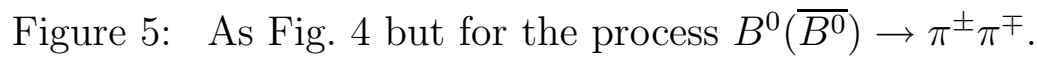




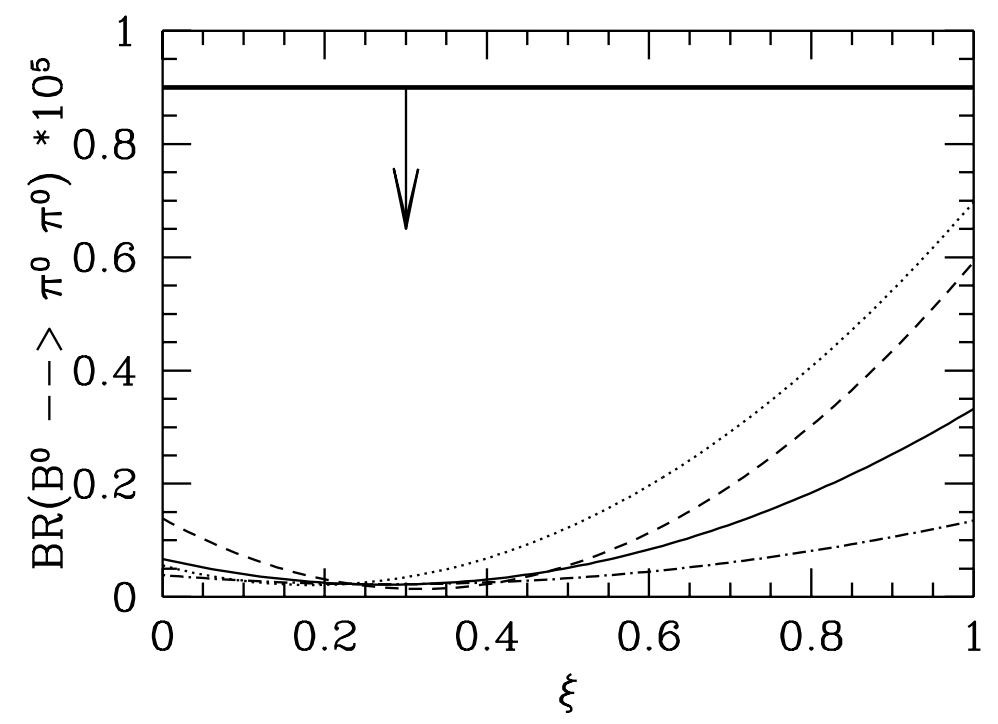

Figure 6: As Fig. 团 but for the process $B^{0}\left(\overline{B^{0}}\right) \rightarrow \pi^{0} \pi^{0}$.

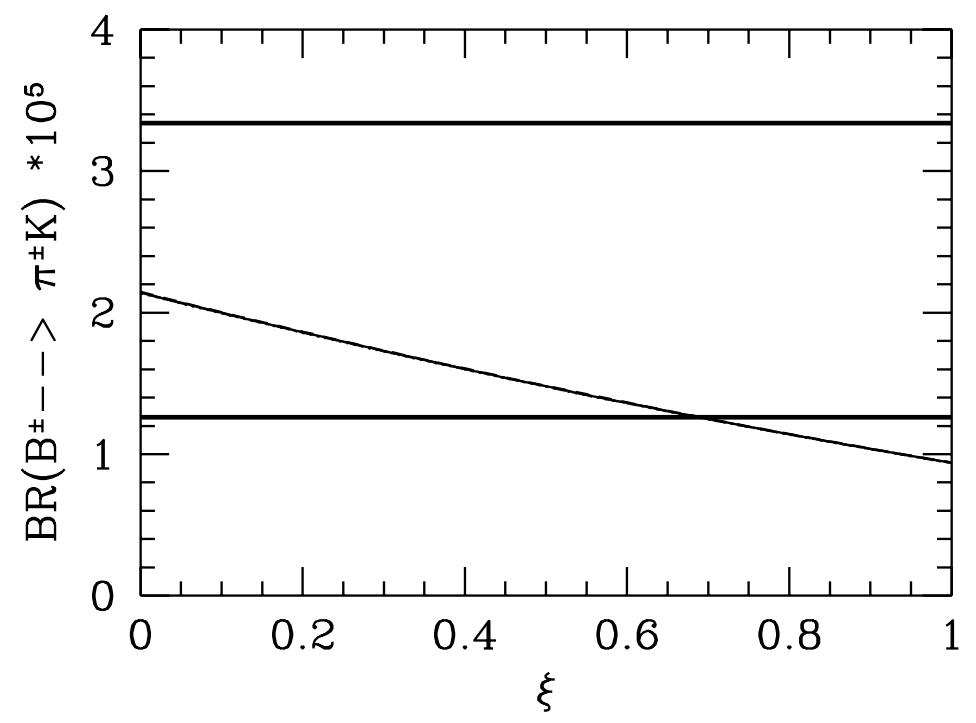

Figure 7: Branching ratio for $B^{ \pm} \rightarrow \pi^{ \pm} K$ as a function of $\xi$ for various points in the $(\rho, \eta)$ plane. Solid line: $(\rho, \eta)=(0.05,0.36)$; dotted line: $(\rho, \eta)=(-0.20,0.45)$; dashed line: $(\rho, \eta)=$ $(0.30,0.42)$; dash-dotted line: $(\rho, \eta)=(0.00,0.22)$. The thick solid lines show the CLEO measurement (with $\pm 1 \sigma$ errors). 


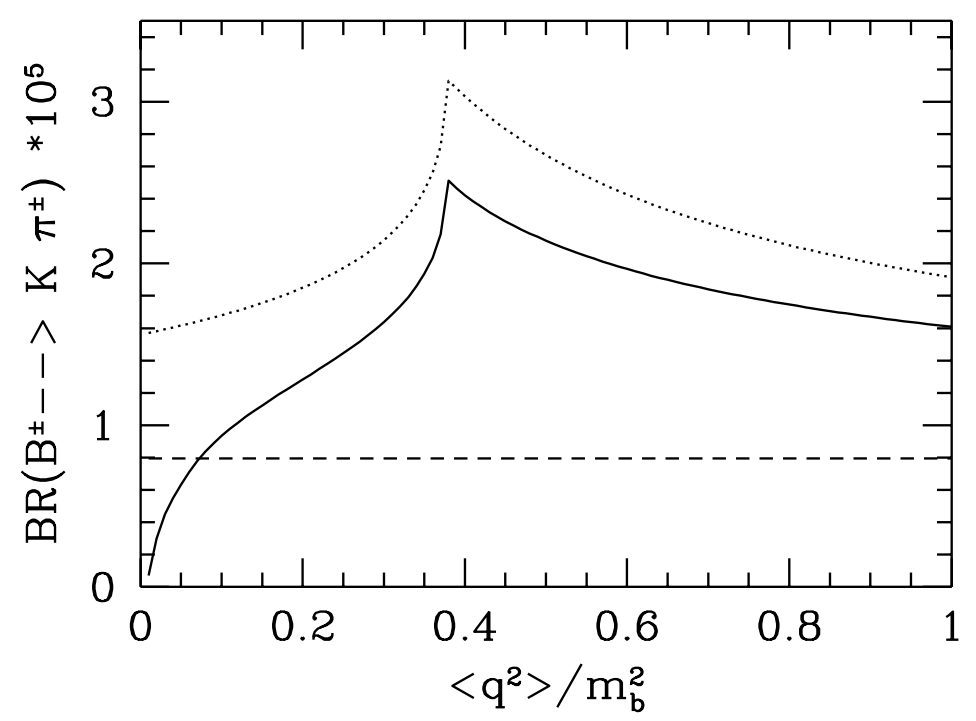

Figure 8: $\mathcal{B}\left(B^{ \pm} \rightarrow \pi^{ \pm} K\right)$ as a function of $\left\langle q^{2}\right\rangle / m_{b}^{2}$. The dashed line corresponds to the LL approximation. The solid line and the dotted line correspond both to the Wilson coefficients evaluated in the NLL approximation; the solid line takes into account the penguin diagrams of all the four-Fermi operators and the tree level matrix element of $O_{8}$, while the dotted line takes into account the penguin diagrams associated with the four-Fermi operators $O_{1,2}^{c}$ only.

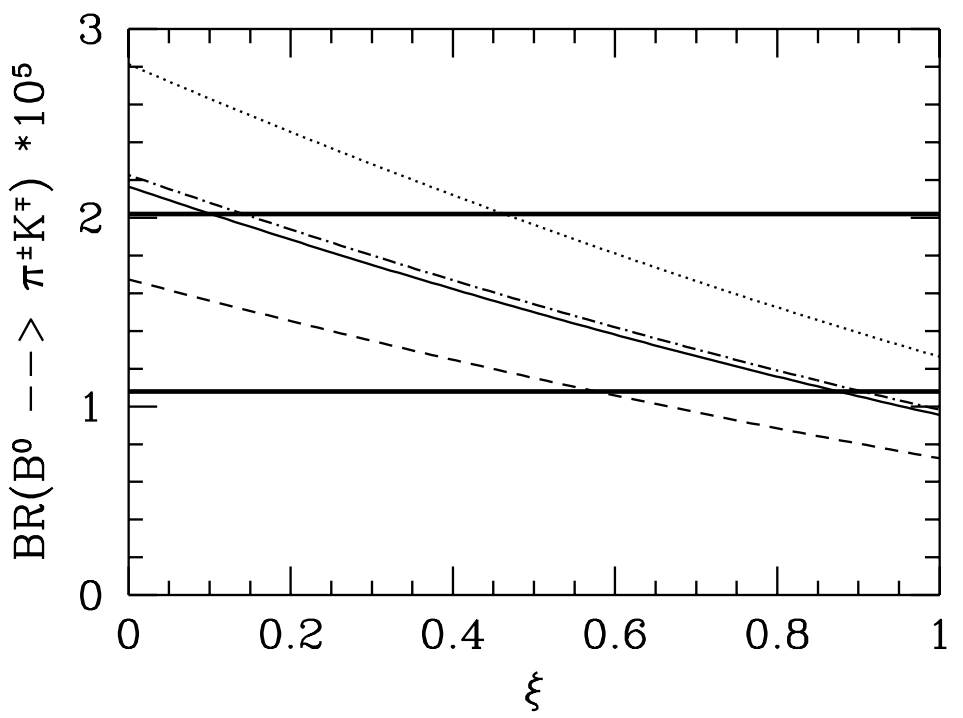

Figure 9: As Fig. 7 but for the process $B^{0}\left(\overline{B^{0}}\right) \rightarrow \pi^{ \pm} K^{\mp}$. 


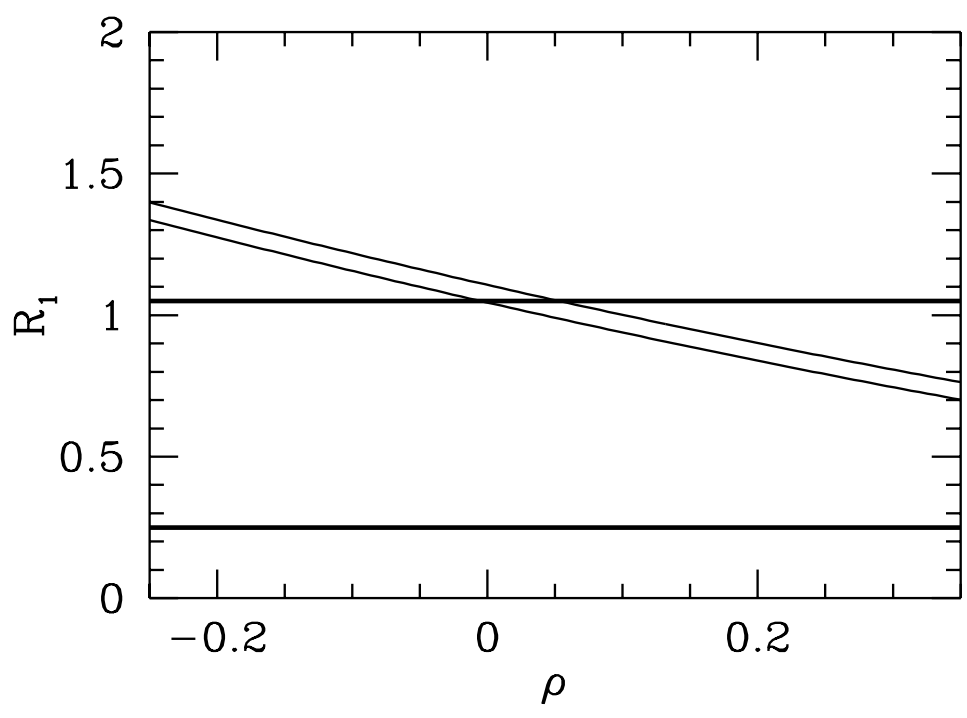

Figure 10: The ratio $R_{1}=\mathcal{B}\left(B^{0}\left(\overline{B^{0}}\right) \rightarrow \pi^{ \pm} K^{\mp}\right) / \mathcal{B}\left(B^{ \pm} \rightarrow K \pi^{ \pm}\right)$as a function of the Wolfenstein parameter $\rho$, for $\eta=0.25$ (lower curve) and $\eta=0.52$ (upper curve). The curves are drawn for $\xi=0$. The horizontal thick solid lines show the CLEO measurement (with $\pm 1 \sigma$ errors).

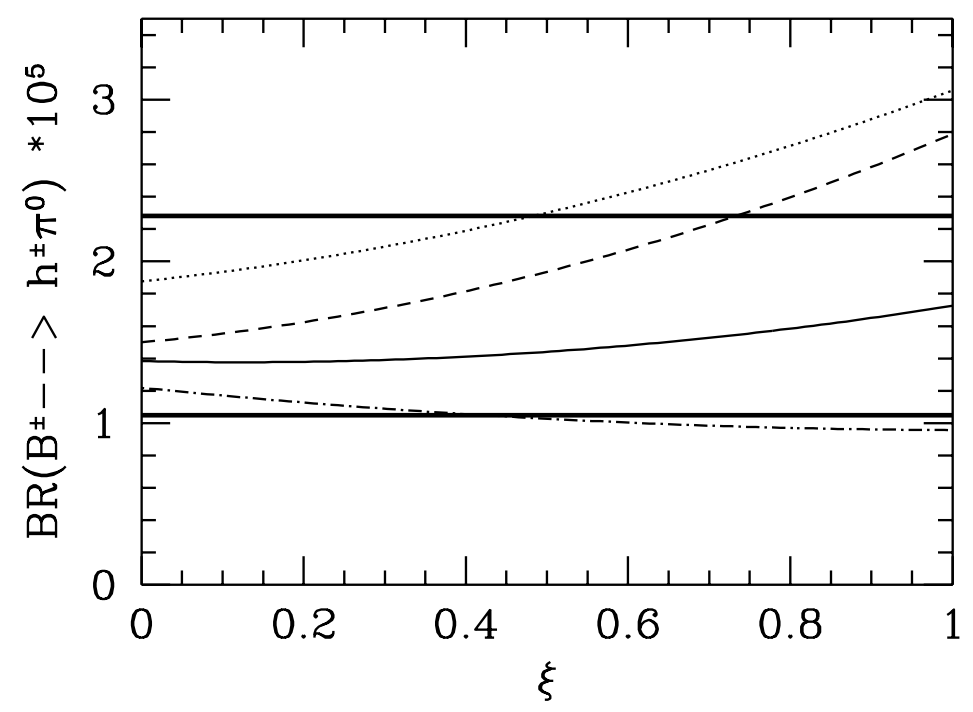

Figure 11: As Fig. 7 but for the process $B^{ \pm} \rightarrow h^{ \pm} \pi^{0}(h=K, \pi)$. 


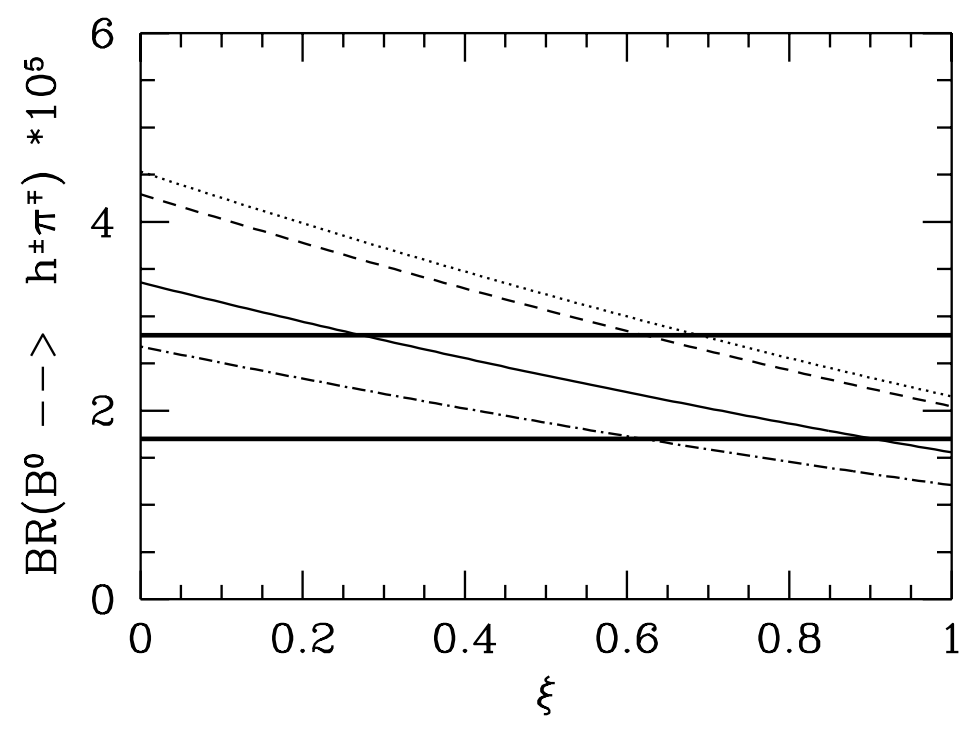

Figure 12: As Fig. 7 but for $B^{0}\left(\overline{B^{0}}\right) \rightarrow h^{ \pm} \pi^{\mp}$.

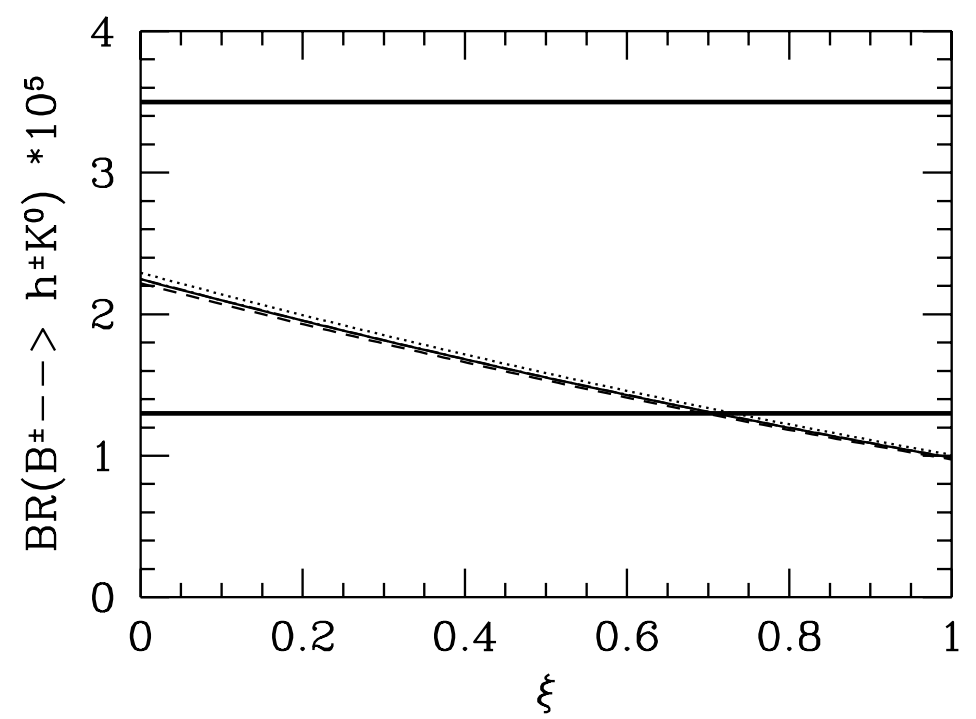

Figure 13: As Fig. 7 but for the process $B^{ \pm} \rightarrow h^{ \pm} K$. 


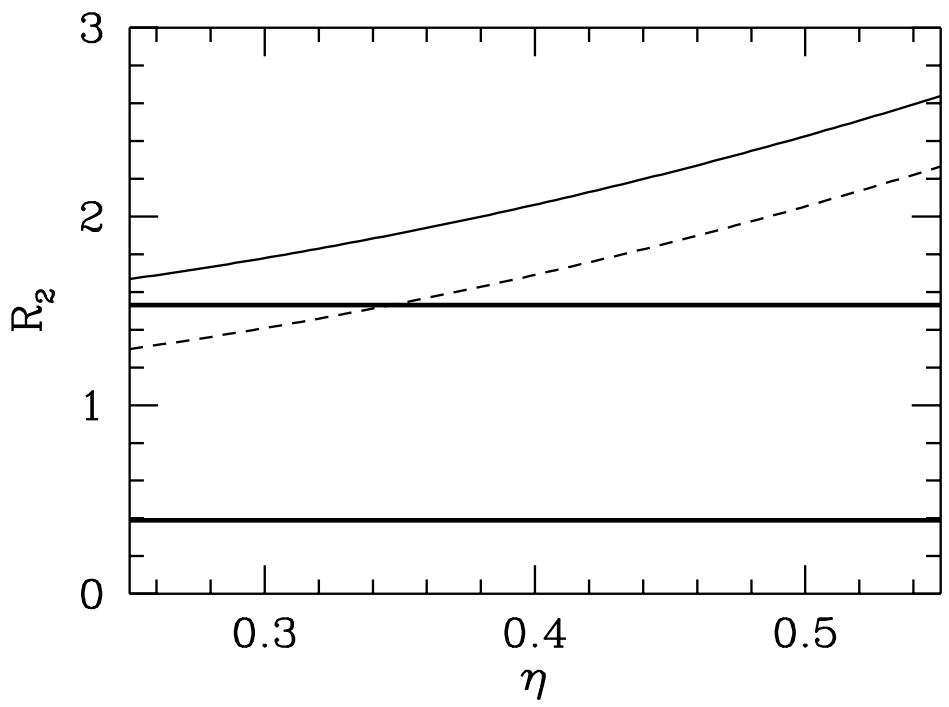

Figure 14: The ratio $R_{2}=\mathcal{B}\left(B^{0}\left(\overline{B^{0}}\right) \rightarrow h^{ \pm} \pi^{\mp}\right) / \mathcal{B}\left(B^{ \pm} \rightarrow K \pi^{ \pm}\right)$as a function of the Wolfenstein parameter $\eta$, for $\rho=0.05$ (dashed curve) and $\rho=0.35$ (solid curve). For $\rho=-0.25$ the corresponding curve is almost identical to the curve for $\rho=0.35$ The curves are drawn for $\xi=0$. The horizontal thick solid lines show the CLEO measurement (with $\pm 1 \sigma$ errors).

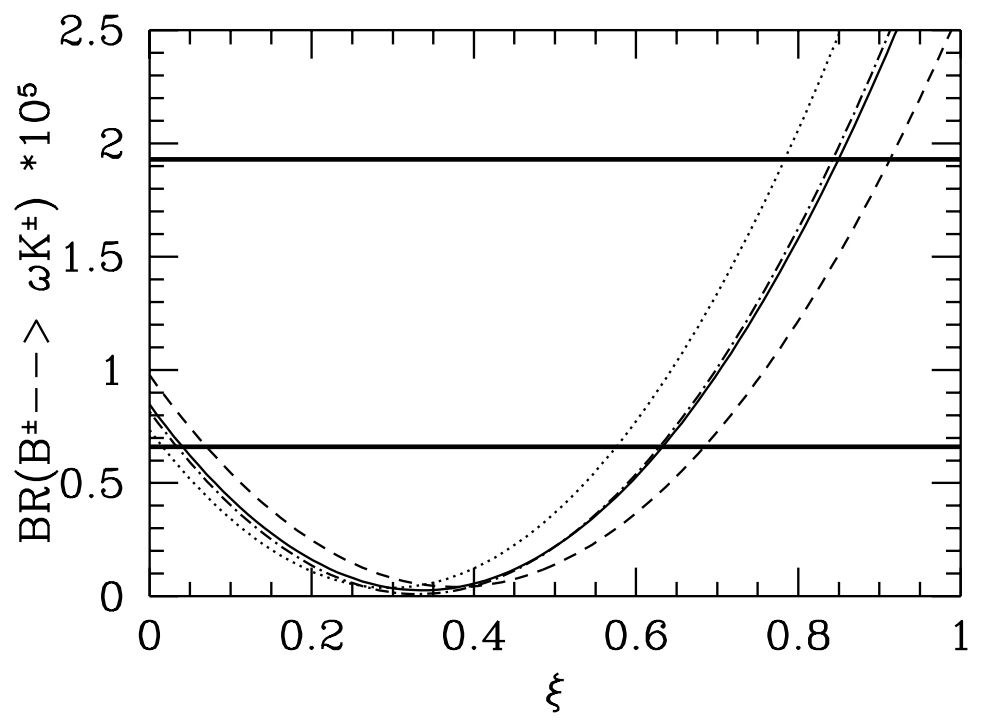

Figure 15: As Fig. 7 but for the process $B^{ \pm} \rightarrow \omega K^{ \pm}$. 


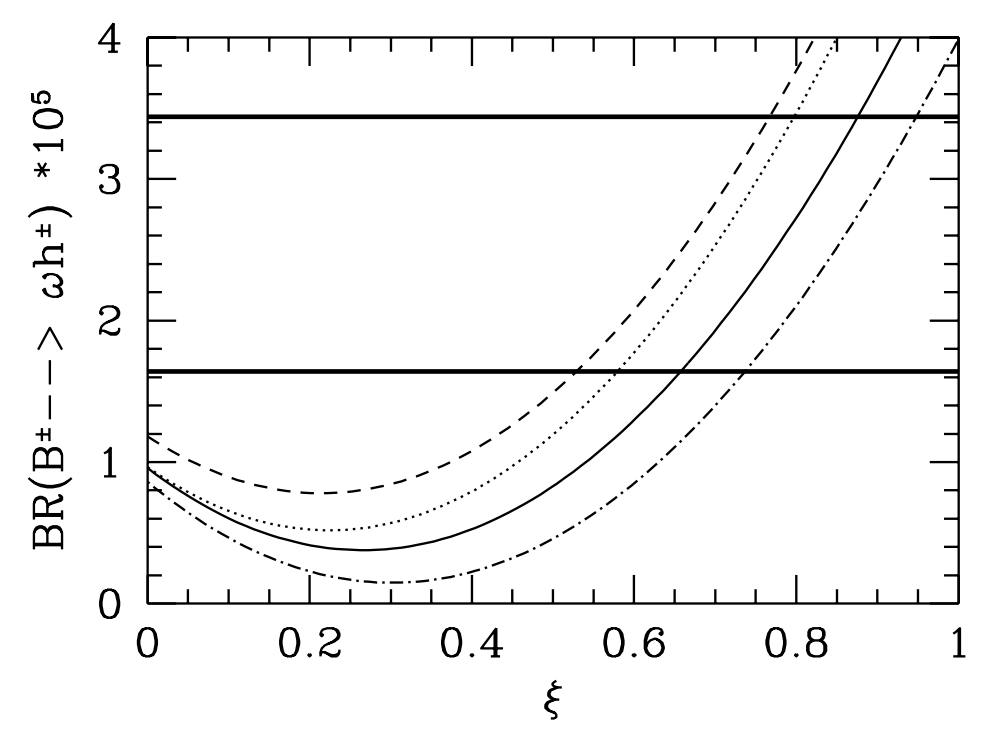

Figure 16: As Fig. 7 but for the process $B^{ \pm} \rightarrow \omega h^{ \pm}$.

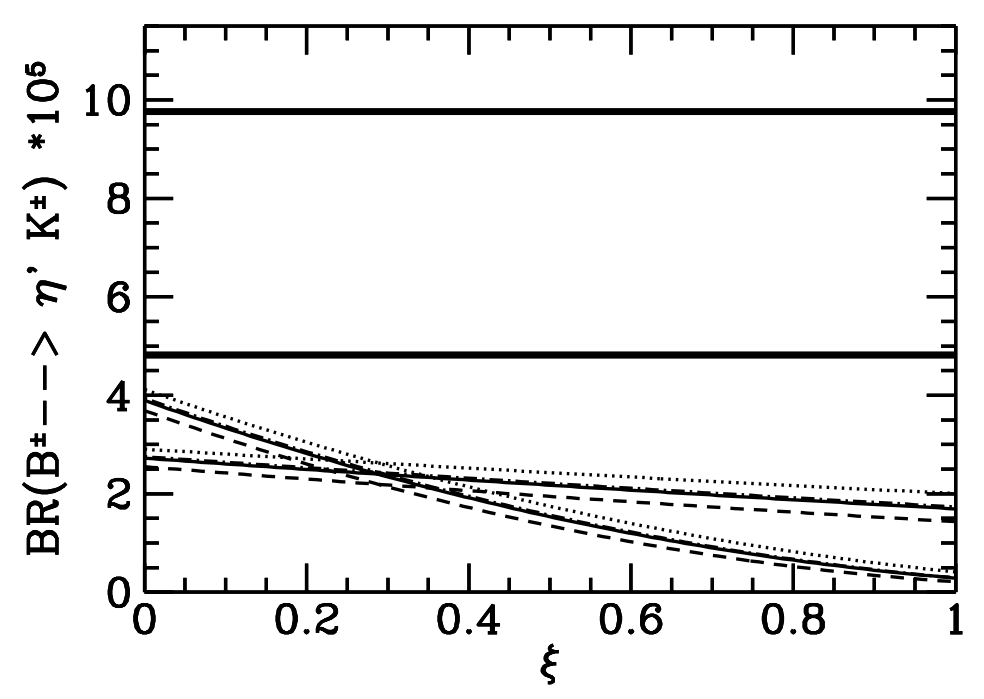

Figure 17: Branching ratio for $B^{ \pm} \rightarrow \eta^{\prime} K^{ \pm}$as a function of $\xi$ for the same points in the $(\rho, \eta)$ plane as in Fig. 7. The upper (lower) set of curves close to $\xi=0$ corresponds to the positive (negative) solution for $f_{\eta^{\prime}}^{(c)}$. The horizontal thick solid lines show the CLEO measurement (with $\pm 1 \sigma$ errors). 


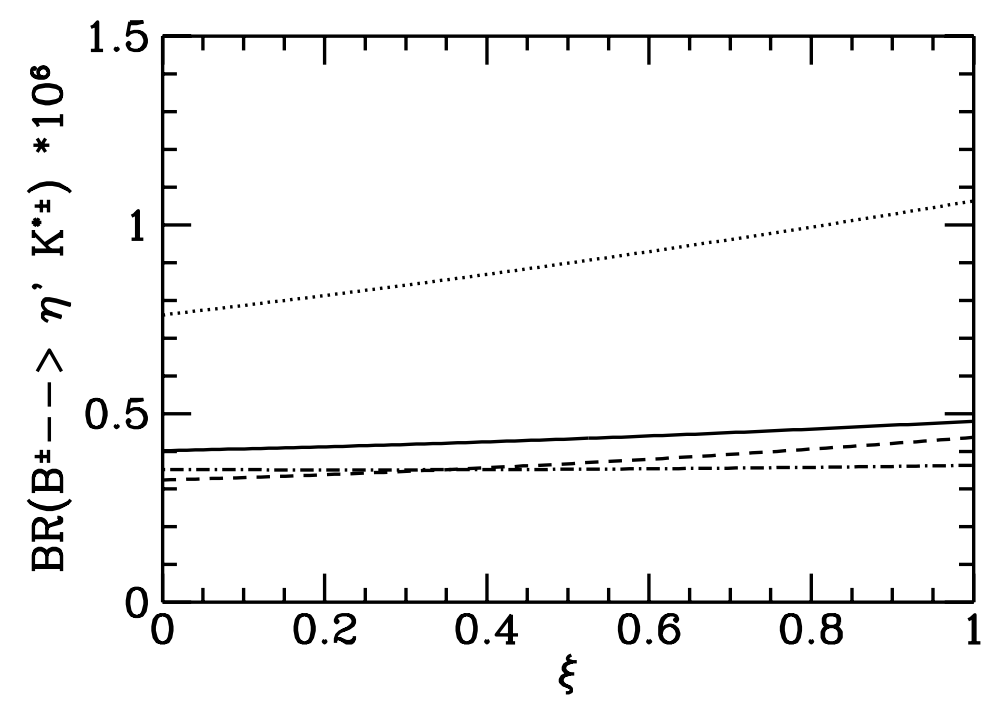

Figure 18: Branching ratio for $B^{ \pm} \rightarrow \eta^{\prime} K^{ \pm *}$ as a function of $\xi$ for the same points in the

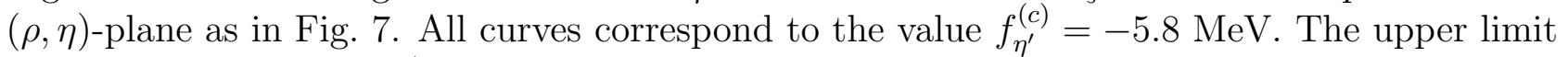
from CLEO is $2.9 \times 10^{-4}$ at $90 \%$ C.L..

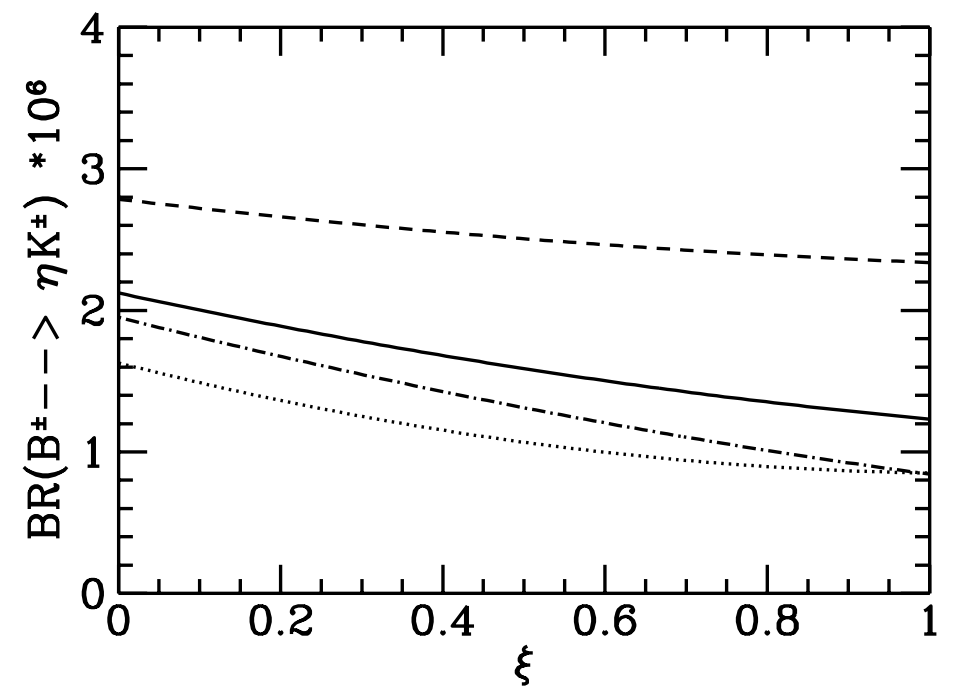

Figure 19: Branching ratio for $B^{ \pm} \rightarrow \eta K^{ \pm}$as a function of $\xi$ for the same points in the $(\rho, \eta)$-plane as in Fig. $\mathrm{Q}$. All curves correspond to the value $f_{\eta}^{(c)}=-0.93 \mathrm{MeV}$. The upper limit from CLEO is $8 \times 10^{-6}$ at $90 \%$ C.L.. 


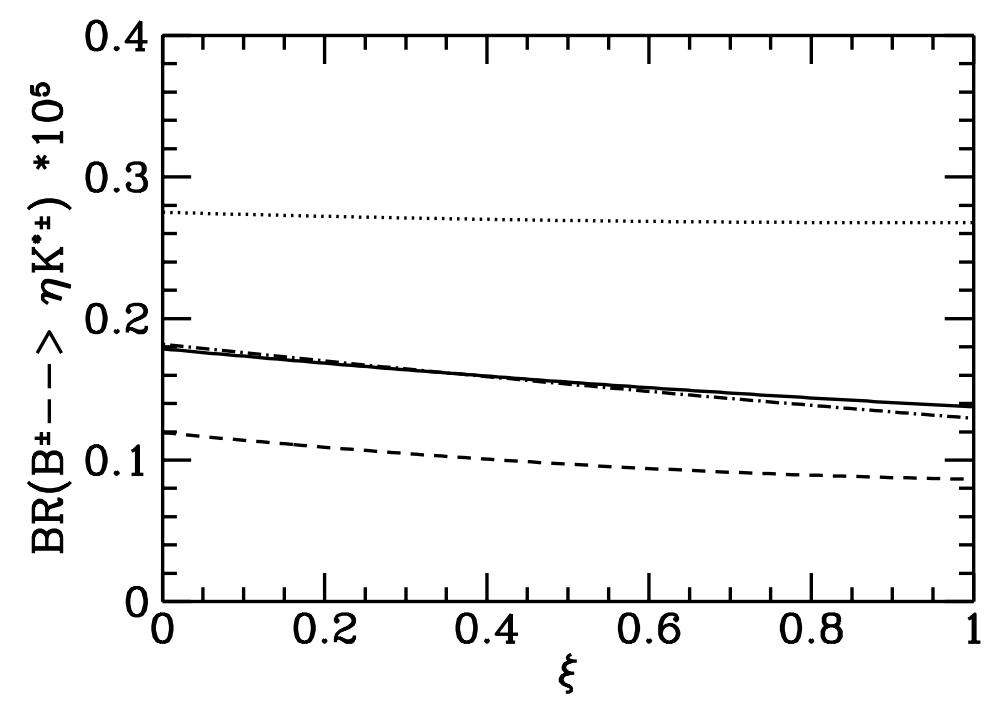

Figure 20: Branching ratio for $B^{ \pm} \rightarrow \eta K^{ \pm *}$ as a function of $\xi$ for the same points in the $(\rho, \eta)$-plane as in Fig. 1 . All curves correspond to the value $f_{\eta}^{(c)}=-0.93 \mathrm{MeV}$. The upper limit from CLEO is $2.4 \times 10^{-4}$ at $90 \%$ C.L..

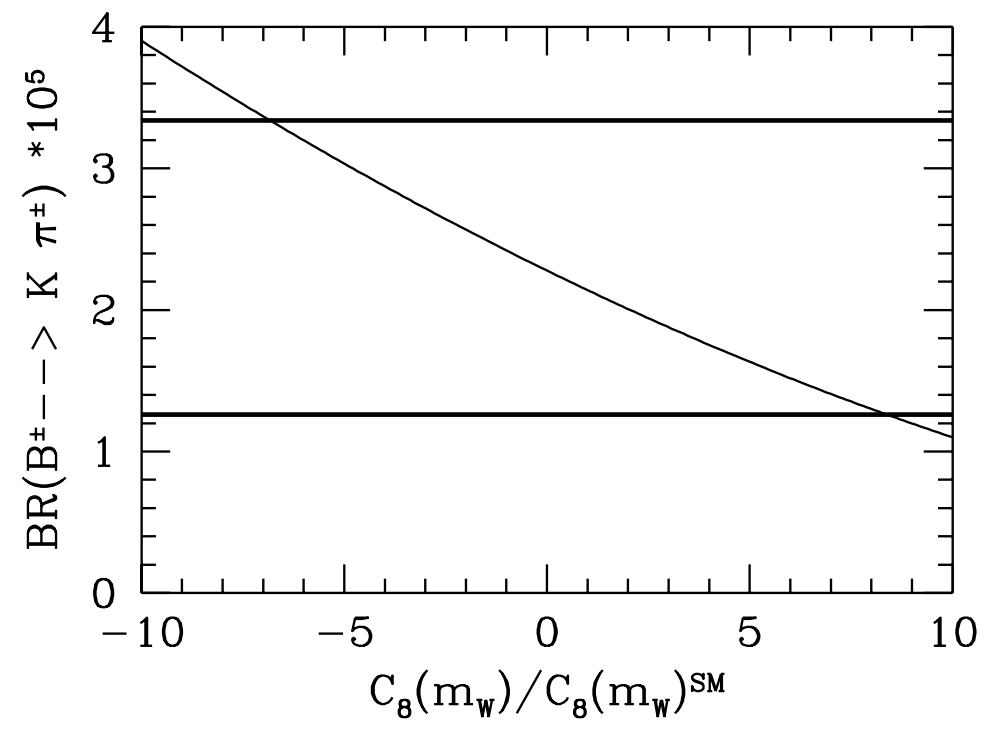

Figure 21: $\mathcal{B}\left(B^{ \pm} \rightarrow K \pi^{ \pm}\right)$as a function of $C_{8}\left(m_{W}\right) / C_{8}^{S M}\left(m_{W}\right)$, where $C_{8}\left(m_{W}\right)$ is the Wilson coefficient of $O_{8}$ including new physics, while $C_{8}^{S M}\left(m_{W}\right)$ is the standard model value. We use $\xi=0$ and $(\rho, \eta)=(0.05,0.36)$. 